\title{
Preparation, characterization and application of the nanocomposite PCL-PEG-PCL / Bentonite-TBHSA to the removal of methylene blue (MB) dye: Adsorption, Kinetics, and Isotherm studies
}

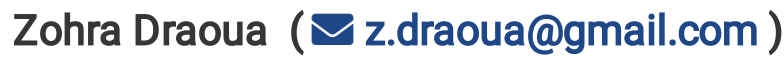

Universite d'Oran 1 Ahmed Ben Bella

Amine Harrane

Mehdi Adjdir

\section{Research Article}

Keywords: Nanocomposites, Adsorption, Methylene blue, Kinetics, Isotherm, Mechanism

Posted Date: April 1st, 2021

DOI: https://doi.org/10.21203/rs.3.rs-363153/v1

License: (c) (i) This work is licensed under a Creative Commons Attribution 4.0 International License.

Read Full License 
Preparation, characterization and application of the nanocomposite PCL-PEG-PCL / Bentonite-TBHSA to the removal of methylene blue (MB) dye: Adsorption, Kinetics, and Isotherm studies

Zohra Draoua $^{1,2}$, Amine Harrane $^{1,3}$ and Mehdi Adjdir ${ }^{4,5}$

${ }^{1}$ Laboratory of Polymer Chemistry, Faculty exact Sciences, Department of chemistry, University Oranl Ahmed Ben Bella, BP 1524 El M'Naouer, 31000 Oran, Algeria.

${ }^{2}$ Department of Civil Engineering, Faculty of Architecture and Civil Engineering, University of Sciences and Technology of Oran Mohamed Boudiaf, USTO-MB, BP 1505, El M'naouer, 31000, Oran, Algeria

${ }^{3}$ Department of Chemistry, FSEI University Abdelhamid ibn Badis Mostaganem, ALGERIA.

3Laboratory of Applied Organic Synthesis, Faculty of Exact and Applied Sciences, University Oran1 Ahmed Ben

Bella, BP 1524 El M'Naouer, 31000 Oran, Algeria

${ }^{5}$ Faculty of Technology, Departement of Engineering Process, University of Saida, Algeria.

Correspondant Authors: Email: zohra.draoua@univ-usto 


\begin{abstract}
This study focuses on the synthesis, characterization, and application of amphiphilic PCLPEG-PCL/Bentonite-TBHSA (A2). The prepared of A2 nanocomposite was prepared from Algerian Bentonite modified by the intercalation of tetrabutylammonium hydrogen sulfate (TBHSA) (A1), were characterized by different techniques including Fourier transform infrared spectroscopy (FTIR), X-ray diffraction(XRD), (TEM),(DSC) and thermogravimetric analysis (TGA ). The removal efficiency of of methylene blue, from aqueous solutions.

The effects of the initial $\mathrm{pH}$ of a solution, contact time, and nanocomposite mass on the adsorption efficiency were investigated. Pseudo-first/second-order isotherms were applied to determine the efficiency of nanocomposite solid. The experimental data fitted well with the pseudo-second-order model for MB dye adsorption. The mass of nanocomposite increased, the adsorption capacity of dye increases to reach an optimal value at $0.13 \mathrm{~g}$ of adsorbent in $\mathrm{pH}=6.8$. The Langmuir isotherm exhibited the best fit, with an adsorption capacity equal to $600 \mathrm{mg} / \mathrm{g}$.
\end{abstract}

KEYWORDS: Nanocomposites, Adsorption, Methylene blue, Kinetics, Isotherm, Mechanism 


\section{Introduction}

Contamination of wastewater by different forms of pollutants is a real problem. Among these pollutants the dyes, which are considered potentially toxic compounds and harmful to the environment [1]. Different industries such as textile, pulp, paper, printing, food, plastic, and tanneries commonly use organic dyes that are aromatic compounds[2]. These dyes can easily be transported in the aqueous environment due to their high solubility in water and therefore can present many serious ecological, environmental and health risks[3]. Various conventional methods have been proposed for the removal of dyes from wastewater including physical and chemical technologies by using phyllosilicate [4,5] zeolites[6], HDLs [7], polymers[8], magadiite [9] and hybrid nanomaterials [10,11], carcinogenic[12-15].The hydrophobic nature of PCL in water systems leads to the stability of its chemical and mechanical properties, favoring its use in aqueous media. On the other hand, PCL leads to the modification of the Bentonite character from hydrophilic to hydrophobic Despite the large investments in biodegradable polymers, it is evident that they are still far from becoming alternatives to the conventional non-degradable polymers for example: Linear high-density polyethene (HDPE), Branched low-density polyethene (LDPE) widely used. In general, the main limitations are due to expensive manufacturing costs and disadvantageous physical properties (eg, poor mechanical and thermal properties, high hydrophobicity, and poor process ability) [16, 17]. Therefore, it seems very necessary to change their chemical nature and/or enhance their performance by nanotechnology. The addition of phyllosilicates into a polymer phase leads to polymer/phyllosilicates composites with enhanced properties. Although there are several elaboration methods developed, the in situ polymerization of monomers mixed with layered silicates seems to be the most suitable way for obtaining either intercalation or the exfoliated structures. This method should have a greater impact on properties than micro-composites [18-25]. In recent years there has been a growing interest in the use of clay minerals such as Bentonite, kaolinite, diatomite and fuller's earth as adsorbents not only to separate inorganic but also organic molecules, this is demonstrated by several researchers [26-28]. In the literature, no work has been reported on the use of PCL-PEG-PCL/Bentonite-TBHSA (A2) as an effective dyes adsorbent. In this work, the structural, morphological, thermal properties and application as an efficient adsorbent of A2 have been extensively studied under different adsorption parameters. 


\section{Materials and methods}

\section{Materials}

ع-Caprolactone (grade 99\%),polyethylene glycol(PEG) (Mn: $2000 \mathrm{I}=1.2$ ) and TBHSA have been suplplied by Aldrich Chemicals, Tetrahydrofuran (THF), Dichloromethane and methanol were dried over $\mathrm{MgSO}_{4}$ and distilled. Tetraobuylammoniumhydrogenosulfate was used as purchased from Aldrich. Bentonite: clay, was procured from (Algerian Society of Bentonite) BENTAL.

Methylene blue (MB), is an organic cationic molecule belongs to the family of Xanthines of the chemical formula $\mathrm{C}_{16} \mathrm{H}_{18} \mathrm{C}_{1} \mathrm{~N}_{3} \mathrm{~S}$ [29]. It possesses a molar mass of $319.86 \mathrm{~g} / \mathrm{mol}$ and a solubility of $50 \mathrm{~g} / \mathrm{L}$ and $10 \mathrm{~g} / \mathrm{L}$ at $20^{\circ} \mathrm{C}$ in water and ethanol, respectively. Also, it is known as methylthioninium chloride or bis (dimethylamino) $-3,7$ phenazathionium chloride. The Figure 1. shows structural formula of the MB dye.

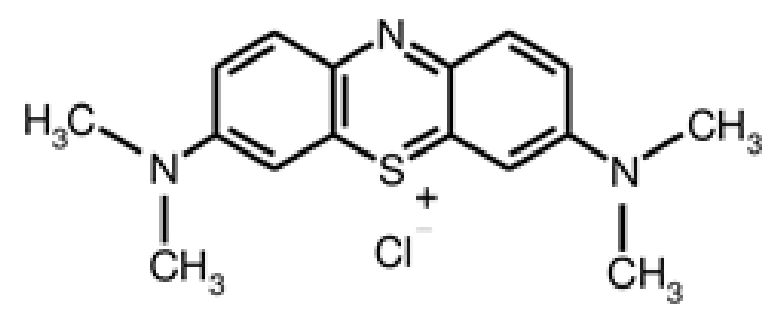

Figure 1. Structural formula of the MB dye

\section{Preparation of Bentonite- $\mathrm{Na}^{+}$}

$100 \mathrm{~g}$ of Bentonite was dispersed in $1 \mathrm{~L}$ of $1 \mathrm{M}$ aqueous solution of $\mathrm{NaPO}_{3}$. The suspension was kept under stirring for 2 hours and then allowed to stand for $24 \mathrm{hr}$ in $2 \mathrm{~L}$ cylinder. The dispersed $<2 \mu \mathrm{m}$ Bentonite-Na clay was collected from the suspension and was then separated by centrifugation under $4500 \mathrm{rpm}$ for $1 \mathrm{~h}$ then dried at $150{ }^{\circ} \mathrm{C}$ under vacuum $[30$ 32].

Organic modification of Bentonite (A1)

Bentonite-Na (10 g) was dispersed in $50 \mathrm{ml}$ distilled water and combined then with $50 \mathrm{ml}$ of $2 \mathrm{M}$ tetrabutylammonium hydrogen sulphate (TBHSA) aqueous solution. The Figure 2. shows structural formula of TBHSA.The mixture was kept under magnetic stirring for $3 \mathrm{~h}$ at room temperature. The composite was then filtered, washed until eliminate the sulphate anions. The 
physisorbed butyl ammonium cations were removed by washing the composite with a preheated water/ethanol mixture50:50. The resulting A1 exchanged with butyl ammonium cations was dried at $50{ }^{\circ} \mathrm{C}$ under vacuum. The organophilic Bentonite produced was dried at $120^{\circ} \mathrm{C}$ and crushed and is called Bentonite-TBHSA (A1).

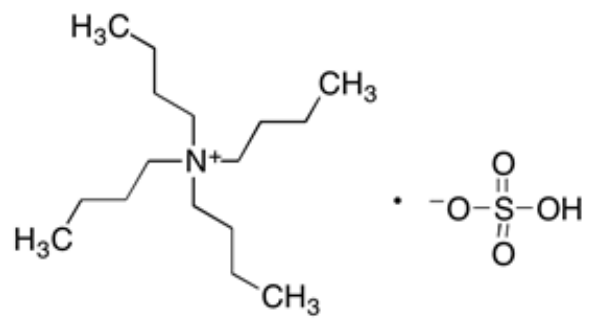

Figure 2. Structural formula of the TBHSA

\section{Characterization of nanocomposites $A 2$}

Structure, morphology and thermal stability of nanocomposites A2 were analyzed by X-ray diffraction (XRD), transmission electron microscopy (TEM), FT-IR, thermogravimetrique Analyse TGA and DSC. XRD diffractograms for pressed powder samples were recorded on a Philips PW 1710 diffractometer using $\mathrm{Cu}-\mathrm{K} \alpha$ radiation $(\lambda=1.5406 \AA)$. dspacings were calculated according to Bragg's law. TEM images were performed with a Philips CM100 apparatus using an acceleration voltage of $100 \mathrm{kV}$. Ultrathin sections of nanocomposites (80 $\mathrm{nm}$ thick) were prepared at $-130{ }^{\circ} \mathrm{C}$ with a Reichert-Jung Ultracut 3E, FC4E ultracryomicrotome equipped with a diamond knife. Because of the large difference in the electron density between silicate and polymer, no selective staining was required to observe the internal structure [33]. Diferential Scanning Calorimetry (DSC) was performed under nitrogen flow $\left(75 \mathrm{~cm}^{3} / \mathrm{min}\right)$ at a heating rate of $10{ }^{\circ} \mathrm{C} / \mathrm{min}$ from room temperature to $200^{\circ} \mathrm{C}$ with a Perkin Elmer Instrument DSC6 thermal analyser. Fourier Transform infrared (FTIR) spectra were recorded between 400 and $4000 \mathrm{~cm}^{-1}$ on Perkin -Elmer B25 spectrometer. Thermal analysis (TG) was performed in air and al the samples were heated to $1000^{\circ} \mathrm{C}$ at heating rate of $10^{\circ} \mathrm{C} \mathrm{min}^{-1}$. 


\section{Adsorption experiments}

The experimental study dye adsorption was carried out in synthetic dilute solutions of methylene blue in batch, by dissolving a specific adsorbent mass in a known concentration solution of $\mathrm{MB}$, during a set time. After this time, the liquid phase is recovered and filtered, and the dye residual concentration was determined from the absorbance analysis (A) which were followed using UV-Visible spectrophotometer, type $2401 \mathrm{PC}$, at $\lambda_{\max }=675 \mathrm{~nm}$. The adsorption equilibrium was achieved by varying; initial dye concentration, 1-100 mg /L; contact time, from 0 to $210 \mathrm{~min}$; mass of the adsorbent, from 20 to $355 \mathrm{mg}$; $\mathrm{pH}, 1-10.2$ and ambient temperature of $25{ }^{\circ} \mathrm{C}$. The percentage of the discoloration $\mathrm{R}(\%)$ and $\mathrm{q}_{\mathrm{e}}$ amount of dye adsorbed $(\mathrm{mg} / \mathrm{g})$ of the $\mathrm{MB}$ were calculated using the following equations, respectively:

$$
\begin{aligned}
& \text { \% adsorption }=\frac{\left(C_{i}-C_{e q}\right) 100}{C_{i}} \\
& q_{e}=\frac{X}{m}=\frac{\left(C_{i}-C_{e q}\right) V}{m}
\end{aligned}
$$

Where $\mathrm{C}_{\mathrm{i}}$ is the initial concentration $(\mathrm{mg} / \mathrm{L}), \mathrm{C}_{\mathrm{eq}}$ is the equilibrium concentration $(\mathrm{mg} / \mathrm{L}), \mathrm{V}$ is the volume of the solution $(\mathrm{L}), \mathrm{m}$ is the mass of adsorbent used $(\mathrm{g})$, and $\mathrm{X}$ number of grams of MB adsorbed (mg).

\section{Results and discussion}

\section{Characterization of adsorbents}

The XRD patterns of Na-Bentonite, TBHSA - Bentonite (A1), and nanocomposite (A2) are shown in Figure 3. The powder XRD pattern of Bentonite-Na was assigned to the typical pattern of layer aluminosilicate Bentonite material with several (001) reflections corresponding to basal spacing of $12.86 \AA$. This pattern compares well with the experimental pattern of Na-Bentonite reported in the Powder Diffraction File under the JCPDS Card No. 003-0019; is in very good agreement with earlier works [10,30,31]. After intercalation with TBHSA, the basal spacing, d001, of the resulting product becomes $14.56 \AA$; indicating the successful of the intercalation method. Indeed, we can assumed that the TBHSA is intercalated as monolayer within the Bentonite layers, this is because of its alkyl chains which 
are very short ( 4 carbons) The d001 of the Bentonite TBHSA (A1) seems to be highly increased after in-situ copolymerisation by caprolactone and ethylene glycol to reach $20.01 \AA$. Based on the high increase of basal spacing after in-situ copolymerisation from $12.86 \AA$ to $20.01 \AA$, we can assume that copolymers are arranged in the interlayer space with paraffin type structure.

The XRD analyses show that, the elaboration of the amphiphilic nanocomposite A2 Figure 3, we observe the decrease of the main peak intensity which characterizes the Bentonite in the region of the small angles for the two samples, indicating the formation of a partially exfoliated/ partially intercalated structure[19,20,27].

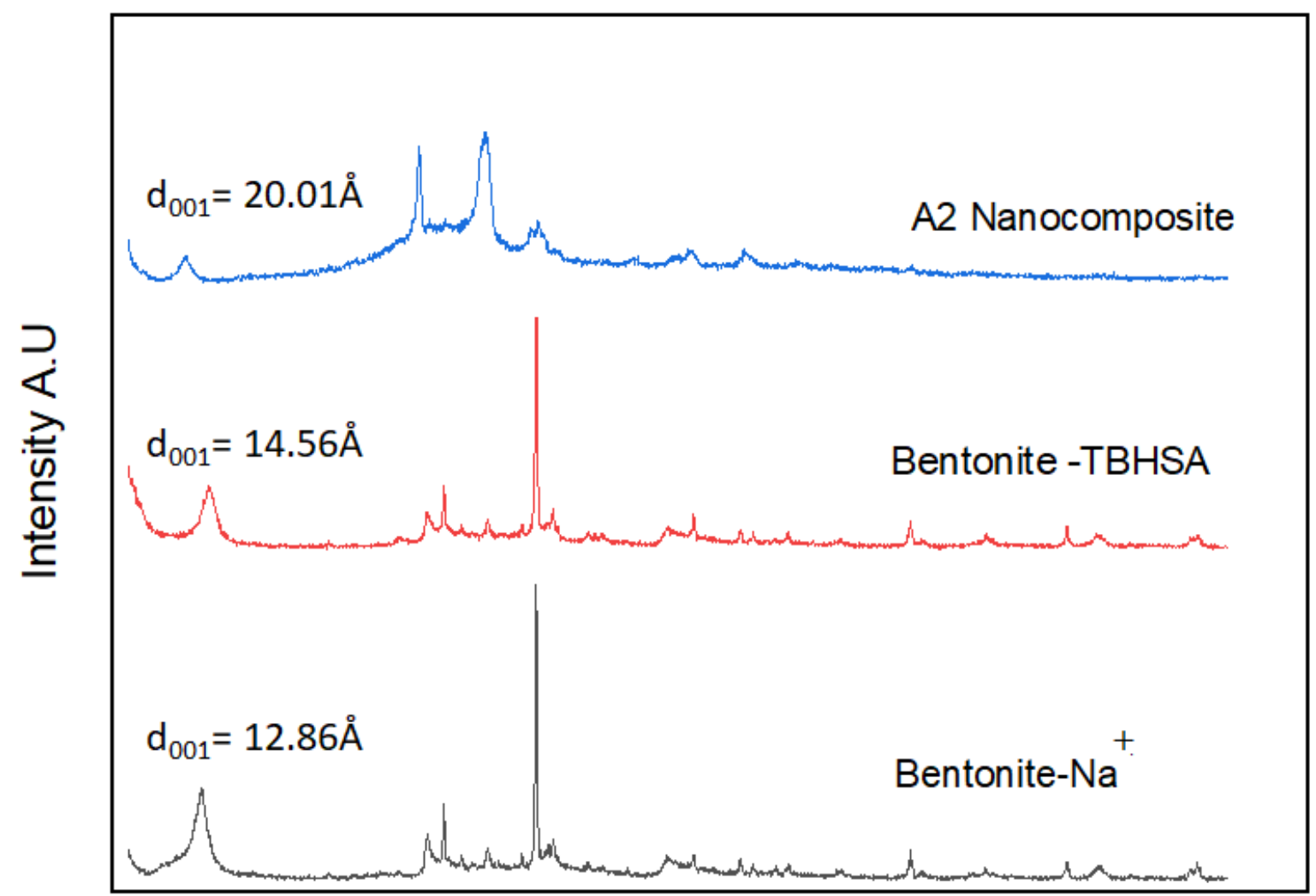

$2 \theta$

Figure 3. XRD patterns of Bentonite- $\mathrm{Na}^{+}$, Bentonite -TBHSA, and A2 Nanocomposite . 


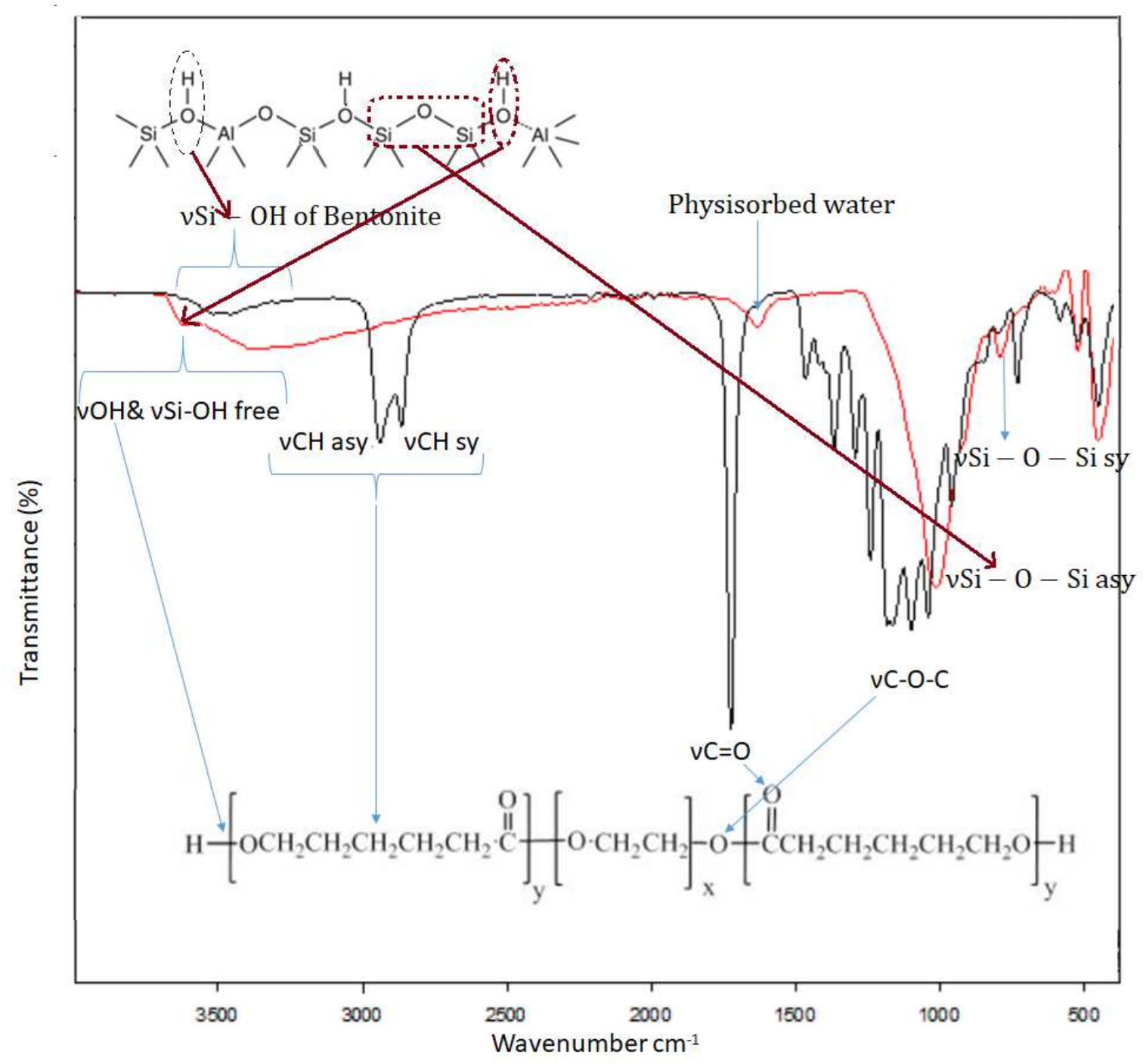

Figure 4. Exhibits the IR functional bands of Bentonite- $\mathrm{Na}^{+}$and Nanocompsite A2.

The FT-IR spectra of Bentonite $-\mathrm{Na}$ and nanocomposite A2 are displayed in Figure4. The results demonstrated that the absorption band at $3619 \mathrm{~cm}^{-1}$ is related to $\mathrm{OH}$ groups either of PCL blocks or to isolated Si-OH of Bentonite [34, 35]. The broad absorption band ranged between $3536-3395 \mathrm{~cm}^{-1}$ is attributed to the vibrations of $\mathrm{OH}$ groups implied in strong interlayer hydrogen bands, $\mathrm{Si}-\mathrm{OH}$, as well as to the interlayer water molecules. The peak of C-H stretching appeared at $2947 \mathrm{~cm}^{-1}$ and $2877 \mathrm{~cm}^{-1}$, which proved the existence of PCL blocks in the copolymer. A Stretching vibration of the ester carbonyl group $(\mathrm{C}=\mathrm{O})$ of PCL blocks appeared at $1736 \mathrm{~cm}^{-1}$. The bands at $1250-1100 \mathrm{~cm}^{-1}$ are associated with the $\mathrm{C}-\mathrm{O}-\mathrm{C}$ stretching vibrations of the $-\mathrm{OCH}_{2} \mathrm{CH}_{2}$ of PEG blocks. The pic at $1670 \mathrm{~cm}^{-1}$ is attributed to the $\mathrm{OH}$ of water physisorbed. 
The Figure 5 shows the TEM image of the A2 nanocomposite. This observation suggests that the fillers were mostly homogeneously distributed in the PCL-PEG-PCL matrix.

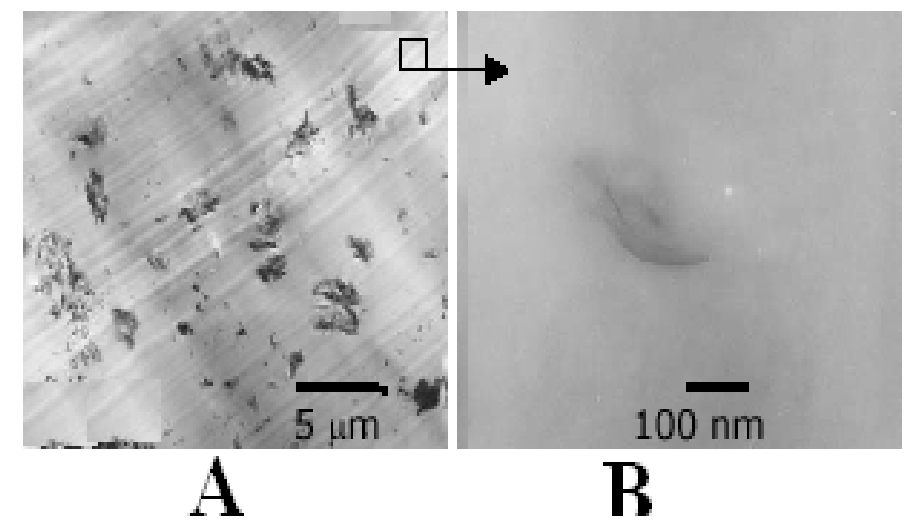

Figure 5. TEM microscopy images for A2 nanocomposite .

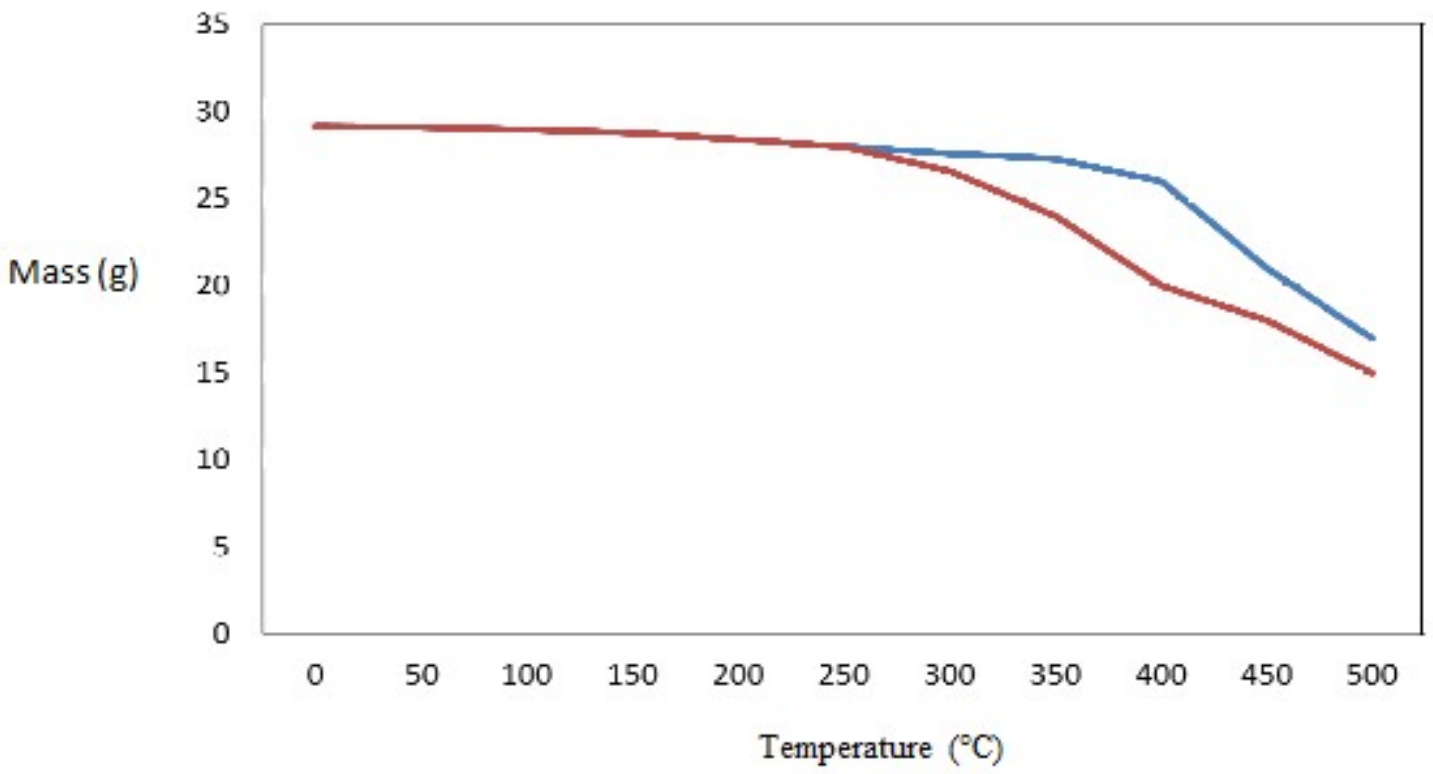

Figure 6. Thermogravimetrique Analyse of Nanocomposite A2- and copolymer pur -

It is observed in figure 6 that at temperatures above $400^{\circ} \mathrm{C}$, the PCL-PEG-PCL/Bentonite mass decreases. This is phenomenon could be assigned to the degradation of the copolymer 
due the high temperature. However, the A2nanocomposite presents an enhancement in the thermal stability from $300^{\circ} \mathrm{C}$ to $400^{\circ} \mathrm{C}$, this improvement is due to the Bentonite framework Figure 6.

The DSC analysis was performed to study the influence of the loading rate of A1/polycaprolactone on the $\mathrm{Tg}$ of the nanocomposite A2 Figure 7. The $\mathrm{Tg}$ of the $\mathrm{A} 2$ nanocomposites appears in the range of $\left(-59^{\circ} \mathrm{C}\right)$ and $\left(-57^{\circ} \mathrm{C}\right)$. This increase of about $15^{\circ} \mathrm{C}$ compared to PCL-PEG-PCL is due to the introduction of clay into the matrix which makes it more rigid. Furthermore, on the thermogram of the A2 nanocomposite, we also observe the presence of a second endothermic peak between (35) and $(50){ }^{\circ} \mathrm{C}$ for different loading rates, which indicates the removal of water physisorbed. The evolution of the crystallinity of the resulted A2 nanocomposites was also studied by DSC measurements. The degree of crystallinity is proportional to the melting enthalpy of the material. As shown in Figure 7, the crystallinity of the resulted nanocomposites, expressed by the melting enthalpy $\Delta H_{m}$ of polycaprolactone segment in the copolymer, increases with high contents of A1.It is known that two processes govern the polymer crystallization, nucleation, which is the mobility of polymer segment to a growing crystal face, and diffusion, the formation of new layers on the crystal [36-40].

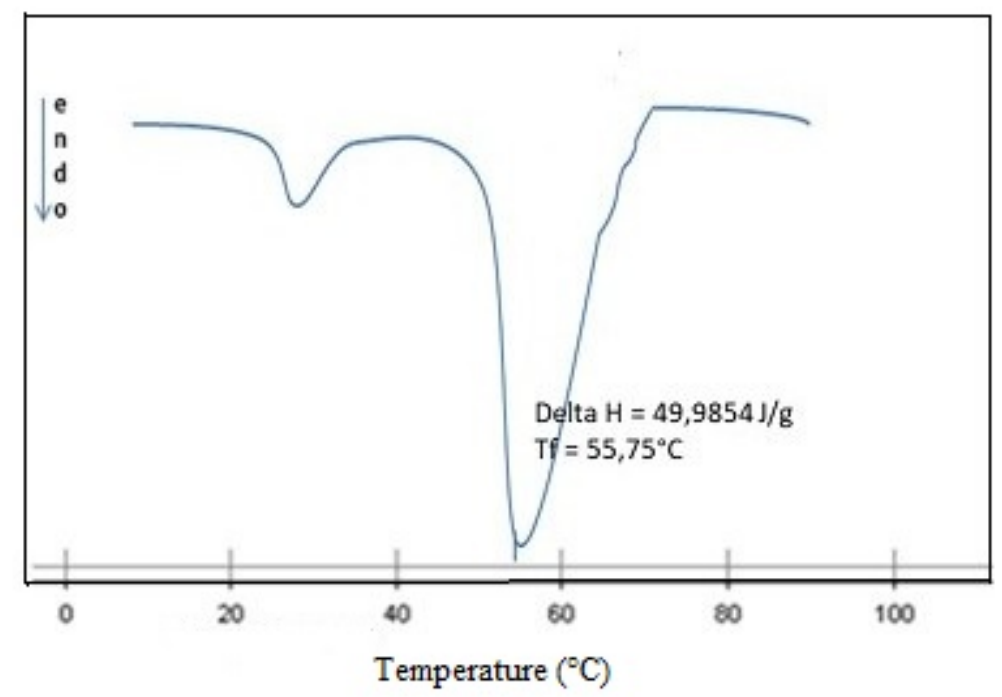

Figure 7. DSC Thermograms of A2 nanocomposites 


\section{$M B$ dye adsorption}

MB dye Adsorption onto different materials is frequently described as a process in order to depend on different parameters specially reaction time which may give many information on the yield and the kinetic adsorption process [41]. In order to investigate the effect of contact time on the elimination of methylene blue, a series of experiments was performed using the following protocol: $0.13 \mathrm{~g}$ of adsorbent in $50 \mathrm{~mL}$ solution with initial concentrations of methylene blue: $10 \mathrm{mg} / \mathrm{L}$ at ambient temperature.

Figure 8 shows the adsorbed amount of MB dye with contact time. The kinetic study of the elimination of MB dye by the adsorbent material shows an increase in the adsorbed amount with the increase in contact time.

It is observed that the maximum adsorption is attained with adsorption capacity of MB dye is around $600 \mathrm{mg} / \mathrm{g}$ within $90 \mathrm{~min}$. In fact, the dye molecules are adsorbed in the first place on the easily accessible sites, a diffusion of these molecules towards the less accessible adsorption sites takes place as the agitation is carried out until reaching the equilibrium value within 90 min. The elimination of MB stabilization (saturation) after the fixed reaction time between this nanocomposite and $\mathrm{MB}$ dye depends on the availability of active and vacant adsorption sites at the surface of the nanocomposite [42]. However, no significant changes were remarked in the removal rate of the MB dye after equilibrium. Similar results have been reported for adsorption of Methylene Blue on activated thermally clays at $500^{\circ} \mathrm{C}$, the adsorption rate was higher at $89 \%$ about $90 \mathrm{~min}[43]$. To evaluate the effectiveness of this material as adsorbents of MB dye, adsorption capacities between this material in this study and various adsorbents. However, the other results were reported for the adsorption of MB. Batch adsorption equilibrium experiments indicated that the adsorbed amount of BM dye by sodium clay was about $330 \mathrm{mg} / \mathrm{g}$ with a rapid initial step and reach equilibrium within 20 $\min [44]$. Also, Dhananasekaran et al studied the adsorption MB dye by $\alpha$-Chitin Nanoparticles [5]. The adsorption process achieved maximum at adsorbed amount about $6.9 \mathrm{mg} / \mathrm{g}$ within 30 $\min$. 


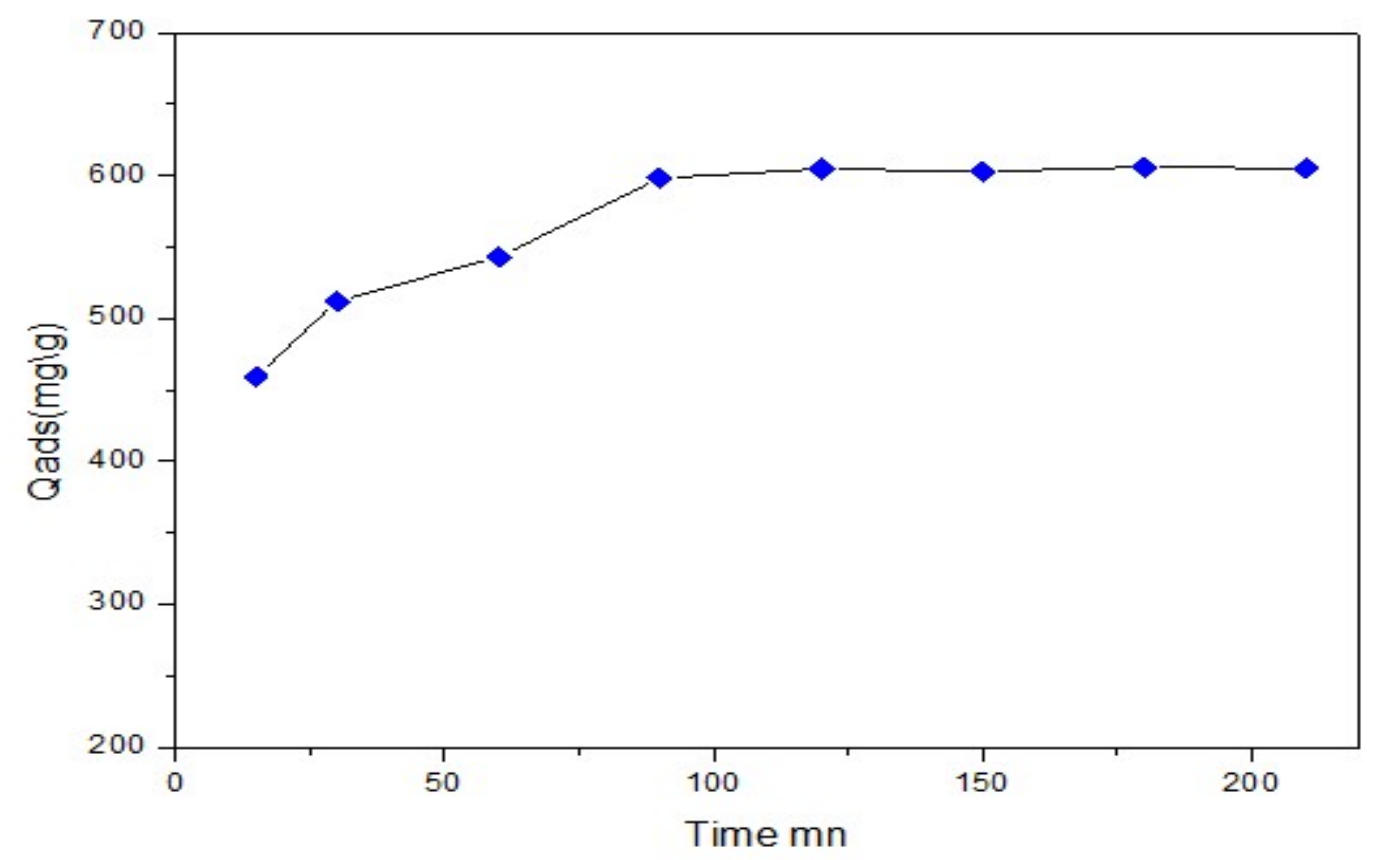

Figure 8. Kinetics of adsorption of $\mathrm{MB}$ on $\mathrm{A} 2$ nanocomposite

\section{Effect of adsorbent mass}

The effect of the adsorbent mass was studied by varying the adsorbent amounts from 0.1 to $0.25 \mathrm{~g}$ for the initial dye concentration of $20 \mathrm{mg} / \mathrm{L}$ at $\mathrm{pH}$ of $\mathrm{MB}$ dye solution within $90 \mathrm{~min}$.

The Figure9 shows that the adsorbed quantities of discoloration increased considerably as the mass of nanocomposite increased, it reaches an optimal value at $0.13 \mathrm{~g}$ then decreases. The optimal amount of the adsorbed dye is very high as it reaches $14.2 \mathrm{mg} / \mathrm{g}$. This result can be explained on the probable availability of more free reactive sites for nanocmposite to interact with dye species. 


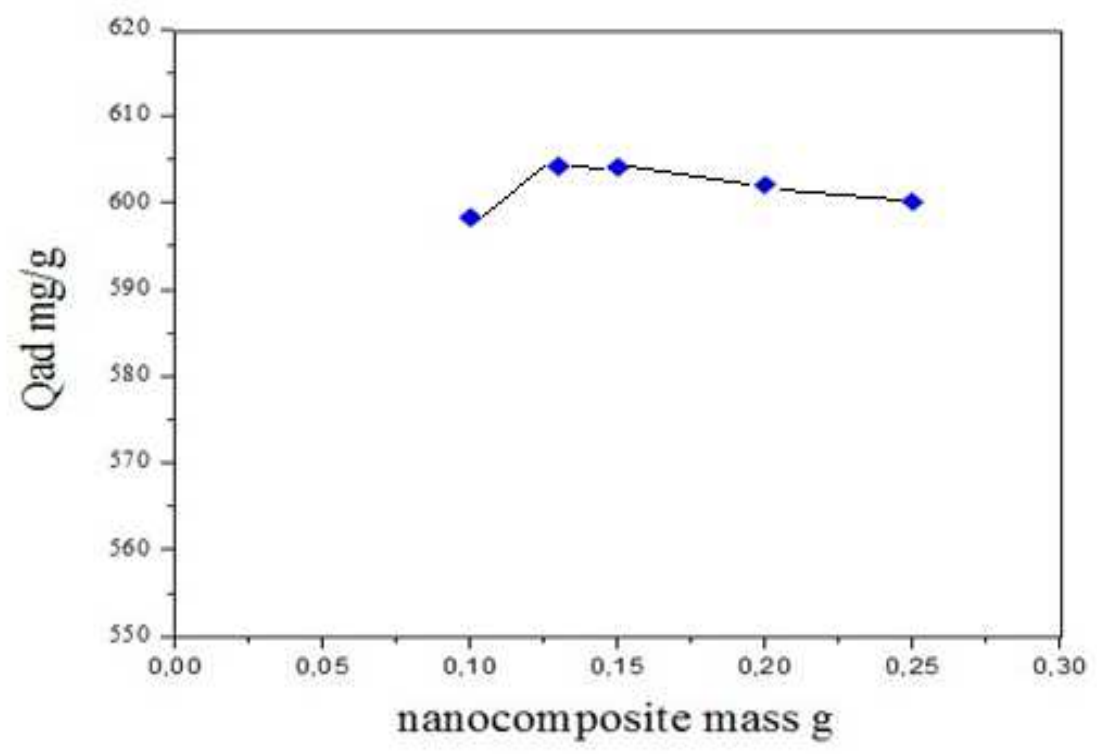

Figure 9. Effect of adsorbent mass on adsorption of MB

\section{Effect of solution pH on MB uptake}

$\mathrm{pH}$ of the aqueous solution, is an important parameter that affects the dye adsorption behavior[5]. Indeed, the adsorption behavior of MB dye on nanocomposite has been studied over a wide range of $\mathrm{pH} 1-10.2$ by adding $\mathrm{HCl}$ or $\mathrm{NaOH}$ to adjust the desired $\mathrm{pH}$ value. The results illustrated at Figure 10 show that the removal rate of the dye is slightly influenced by the $\mathrm{pH}$ changes. However, it can be adsorbed at acidic and basic $\mathrm{pH}$.

The obtained results revealed a rather increase of $\mathrm{MB}$ removal with the $\mathrm{pH}$, reached the maximum from $\mathrm{pH}=4$ to the initial $\mathrm{pH}$ solution of $\mathrm{MB} \mathrm{pH}=6.8$, therefore it decreased slightly with a lowering up to $10 \%$ at basic medium. The maximum adsorption is attained the maximum dye removal efficiency about $98 \%$ at the initial $\mathrm{pH}$ solution of $\mathrm{MB} \mathrm{pH}=6.8$. Nevertheless, the variation of the level of influence of the solution $\mathrm{pH}$ on the $\mathrm{MB}$ dye adsorption efficiency depends on the adsorbent-adsorbate interaction [42]. The dye adsorption was attributed to the hydrophobic-hydrophobic interaction between hydrophobic groups of PCL-PEG-PCL molecules and cationic dye as the carbon chain C16 and the sulfonate groups. Indeed, many investigations were reported using other adsorbents such as: activated carbon from vegetable sponge of cylindrical loofa [45] wheat shells[46] and clay[47] . In an acid medium, the $\mathrm{H}^{+}$ions are very mobile and might occupy the free active sites of the nanocomposite surface or can be also attracted by the different active sites of MB dye namely $\mathrm{S}$ and $\mathrm{N}$ [48]. Nevertheless, there are alternative ions competing between $\mathrm{H}^{+}$ions and $\mathrm{MB}$ dye species. Also, the surface of the nanocomposite based clay becomes positive 
charged which creates more free reactive sites and a combined interaction with MB dye species and this leads to encourage its adsorption effectiveness.

This statement is confirmed by the high removal efficiency at lower $\mathrm{pHs}$. At high $\mathrm{pH}$, the surface of nanocomposite can be deprotonated and typically create negative charge. Thus, it is advantageous that the process is a suitable application for MB dye removal because of its neutral and clean effluent [42]. However, the initial solution $\mathrm{pH}$ of 6.8 was chosen for the rest of the experiments on MB dye adsorption.

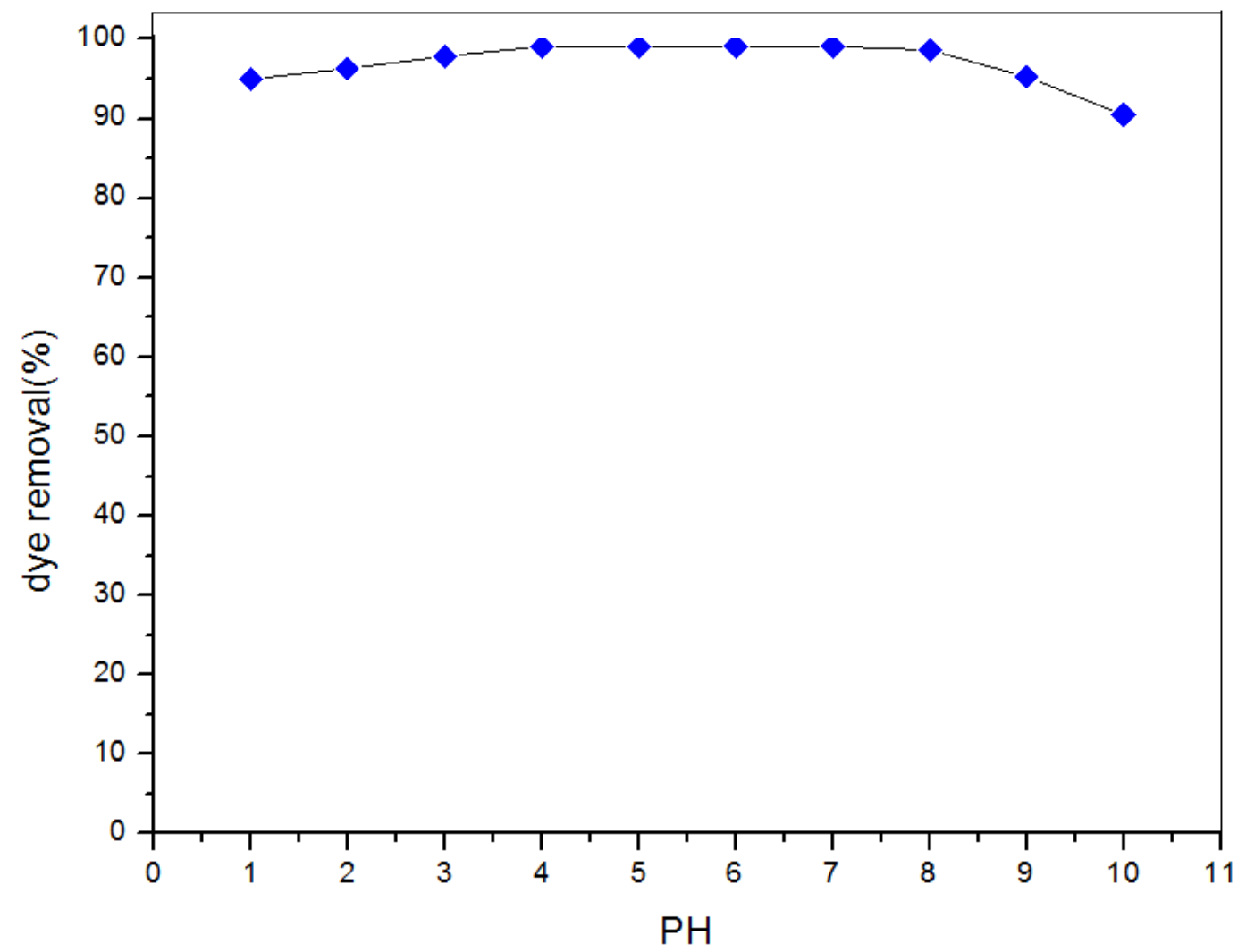

Figure 10. Effect of $\mathrm{pH}$ on adsorption of $\mathrm{MB}$ 


\section{Kinetic adsorption study}

Kinetic study has prime importance in dye adsorption process because it acquire the rate efficiency of adsorption with respect to time and also describe the mechanism of adsorption between the adsorbate and the adsorbent[46] .

The adsorption kinetics was investigated by following of methylene blue concentrations with respect to time. Indeed, two models were applied to describe the adsorption kinetics mechanism of methylene blue. The experimental data were fitted to the Lagergren first-order kinetic model (equation 6) and pseudo second order kinetic model (equation 7) as follow [43]:

$$
\begin{aligned}
& \operatorname{Ln}\left(q_{e}-q_{t}\right)=\operatorname{Ln}\left(q_{e}\right)-\left(K_{1}\right) t \\
& \frac{t}{q_{t}}=\left(\frac{1}{K_{2} q_{e}^{2}}\right)+\left(\frac{1}{q_{e}}\right) t
\end{aligned}
$$

Where $\mathrm{qt}$ and $\mathrm{q}_{\mathrm{e}}$ are the amount of adsorption at time at equilibrium $(\mathrm{mg} / \mathrm{g})$, respectively; $\mathrm{k}_{1}$ is the constant rate of pseudo-first order $(1 / \mathrm{min}) ; \mathrm{k}_{2}$ is the constant rate of pseudo-second order ( $\mathrm{g} /(\mathrm{mg} \mathrm{min})$ ). The application of linear form of the pseudo-second-order equation for the MB dye adsorption onto this nanocomposite is depicted in Figure 11 and Figure 12.The kinetic constant rates $\mathrm{K}_{1}, \mathrm{~K}_{2}$ and the amount of adsorption at equilibrium $\mathrm{q}_{\mathrm{e}}$ were determined from the slope and intercept of the corresponding linear forms, respectively.

The results obtained from Table 1 show that the data kinetic adsorption calculated from pseudo-first order model are not valid to describe the MB dye species mechanism adsorption by A2 nanocomposite. However, it observed also that the pseudo-second order is the mostly suitable to fit the experimental data than pseudo-first order, since its correlation coefficients are higher than those of the first-second order model. However, it was found that the kinetic constant rate $\mathrm{K}_{2}$ was of $106 \mathrm{~g} / \mathrm{mg} . \mathrm{h}$ and correlation coefficient of 0.98 . Indeed, the experimentally adsorbed quantities at equilibrium $\mathrm{mg} / \mathrm{g}$ were found to be in good agreement with those calculated from the second-order reaction kinetics equation $(641 \mathrm{mg} / \mathrm{g})$. These obtained results are in agreement with similar trends previously recorded for MB dye removal by thermally activated clays [43] and activated carbon from vegetable sponge of cylindrical loofa[45]. 


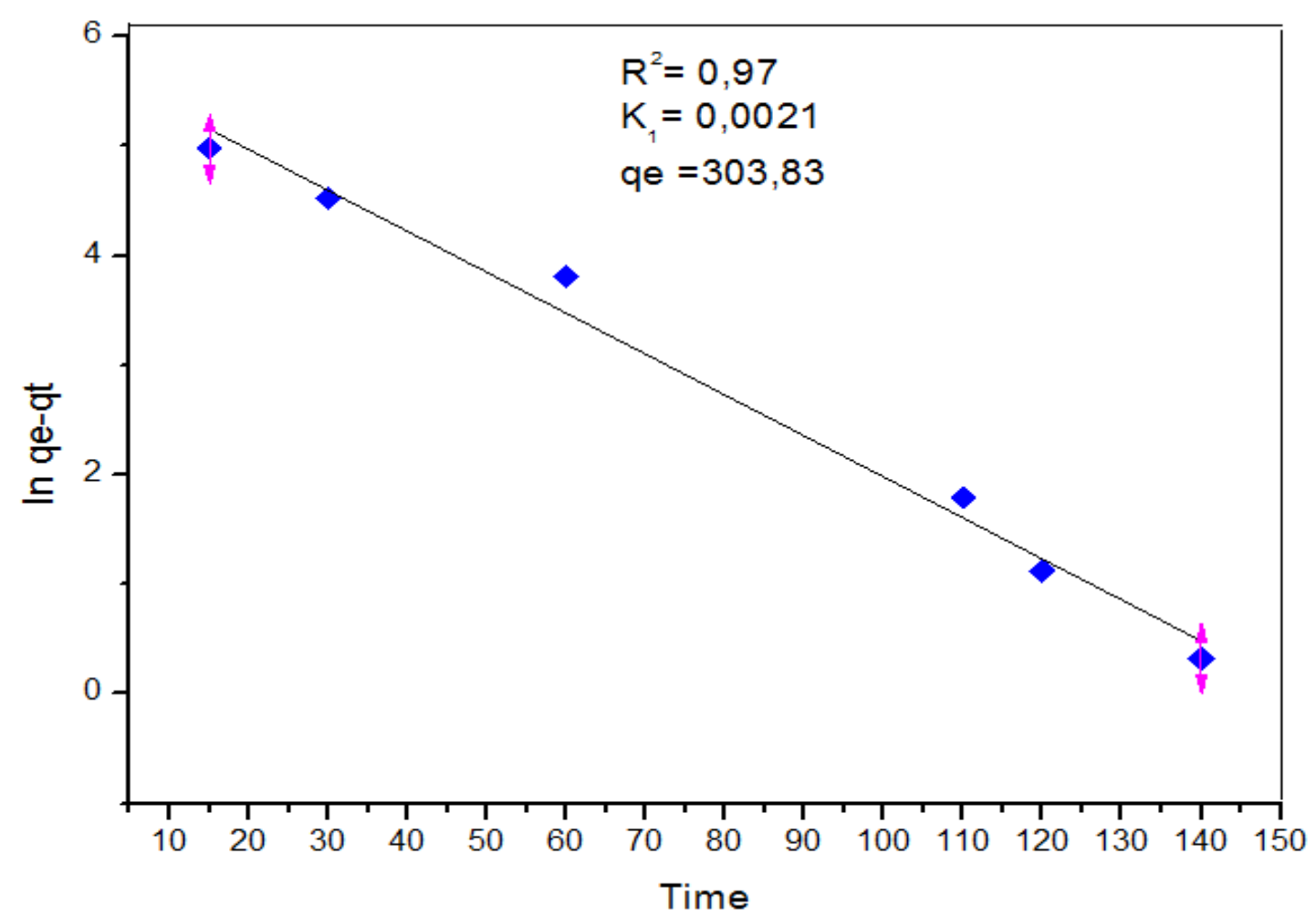

Figure 11. Pseudo-first order for the adsorption of MB on A2 nanocomposite

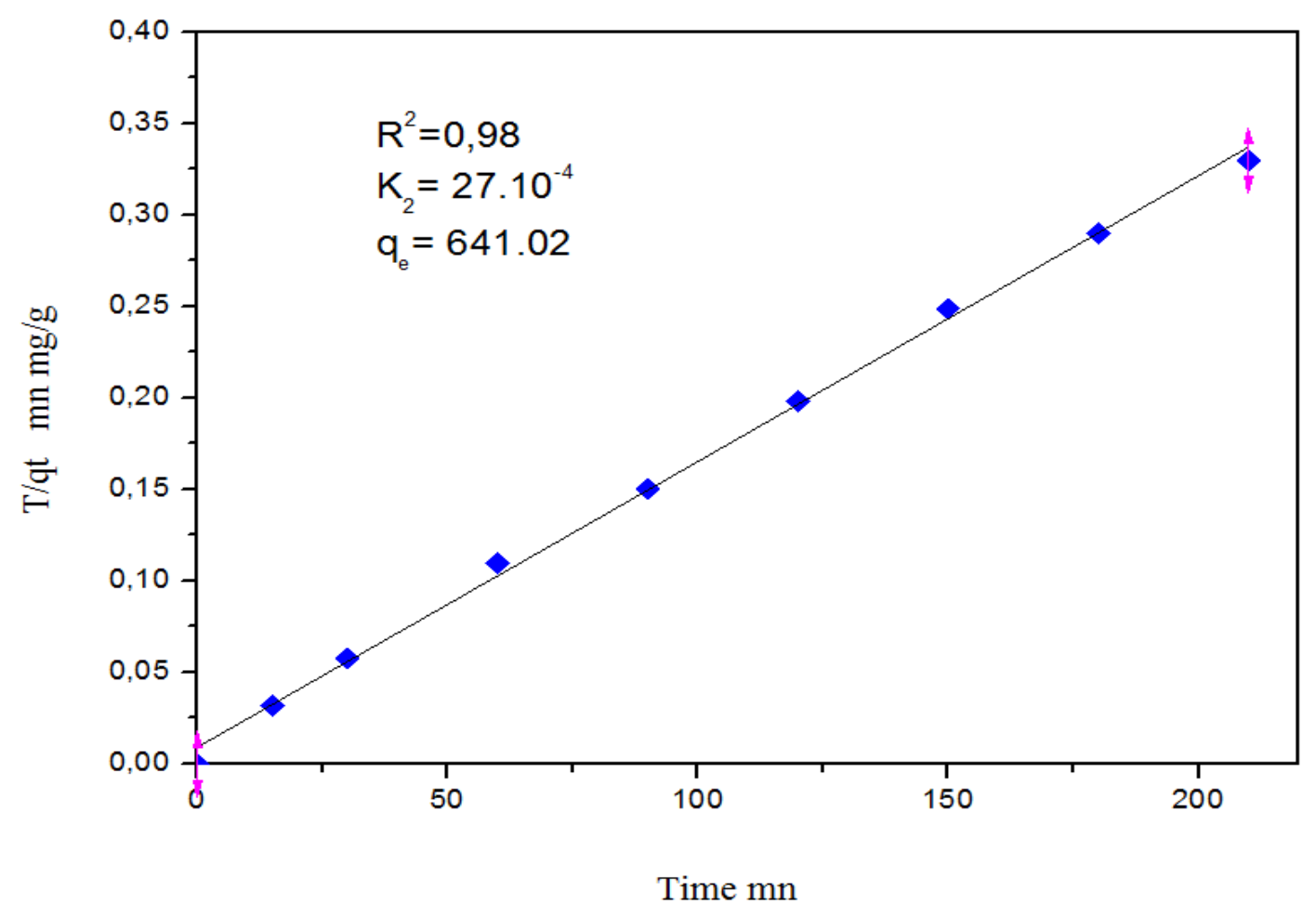

Figure 12. Pseudo-second order for the adsorption of MB onto A2 nanocomposite 
Table 1. Kinetic models for MB dye adsorption onto composite

\begin{tabular}{|c|c|c|c|c|c|c|c|c|}
\hline \multirow{2}{*}{$\begin{array}{c}\text { Adsorbate and } \\
\text { adsorbent }\end{array}$} & \multirow[b]{2}{*}{$\begin{array}{c}\mathrm{C}_{0} \\
(\mathrm{ppm})\end{array}$} & \multirow[b]{2}{*}{$\begin{array}{l}\mathrm{q}_{\mathrm{e}}(\mathrm{mg} / \mathrm{g}) \\
\text { calculée }\end{array}$} & \multicolumn{3}{|c|}{ Pseudo-first order } & \multicolumn{3}{|c|}{ Pseudo-second order } \\
\hline & & & $\begin{array}{l}\mathrm{q}_{\mathrm{e}}(\exp ) \\
(\mathrm{mg} / \mathrm{g})\end{array}$ & $\mathrm{K}_{1}$ & $\mathrm{R}_{1}^{2}$ & $\begin{array}{l}\mathrm{q}_{\mathrm{e}}(\mathrm{exp}) \\
(\mathrm{mg} / \mathrm{g})\end{array}$ & $\mathrm{K}_{2}$ & $\mathrm{R}_{2}^{2}$ \\
\hline $\begin{array}{l}\text { Nanocomposite- } \\
\text { methylen blue }\end{array}$ & 10 & 600 & 303.83 & 0.0021 & 0.97 & 641,02 & $27.10^{-4}$ & 0.98 \\
\hline
\end{tabular}

\section{Adsorption isotherm study}

To evaluate the adsorbed amount capacity of the adsorption isotherm with various initial concentrations of $\mathrm{MB}$ dye ranging from 5 to $30 \mathrm{mgL}^{-1}$. The adsorption isotherm non linear has been determined by plotting the relation of $\mathrm{q}_{\mathrm{e}}$ as a function of $\mathrm{C}_{\mathrm{eq}}$ Figure 13.

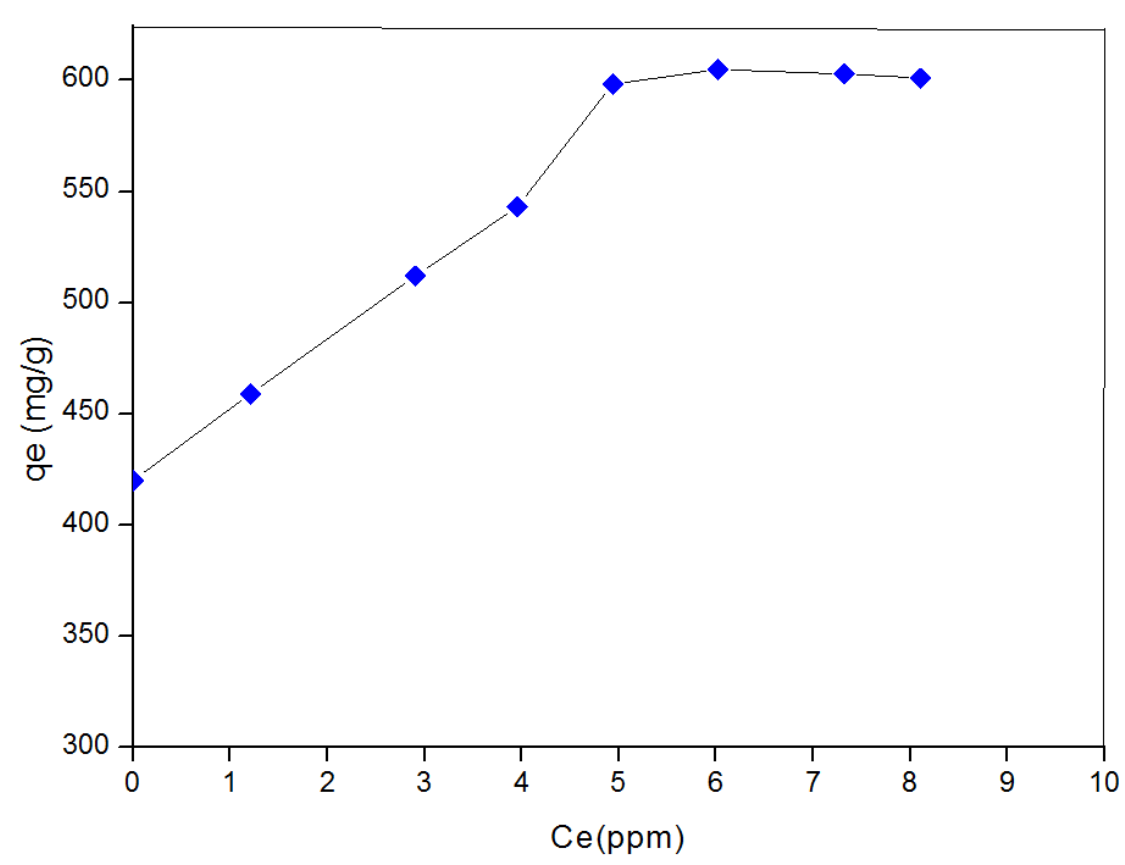

Figure 13. Adsorption isotherm of the $\mathrm{MB}$ onto $\mathrm{A} 2$ nanocomposite

The Figure 13. shows that the adsorption capacity increases with the initial concentration. The recorded highest adsorbed amount at equilibrium of MB dye was observed about 600 $\mathrm{mg} / \mathrm{g}$. 
The isotherm shows almost steady state value indicating the saturation of the adsorbent surface and then formation of a monolayer. These isotherms are of type $\mathrm{L}$ in the classification of Giles and all $[42,49,50]$.

This corroborates with similar findings in the case of methylene blue adsorption on a copper montmorillonite and thermally activated clays $[43,44]$.

Langmuir, Freundlich and Temkin isotherm models were applied to assess the dye adsorption phenomena onto this nanocomposite.

The Langmuir isotherm model is the most widely used to present liquid - solid adsorption which assumes monolayer adsorption, Whereas, Freundlich model is empirical which assumes the heterogeneous adsorption on the surfac[51,52]. However, Temkin model assumes that the decrease in heat of absorption with recovery rate is linear rather than logarithmic $[42,51,35]$.

The linear form of Langmuir, Freundlich and Temkin isotherm models were presented in the Figure14, Figure15 and Figure 16) which can be determined by the following relations [43] .

$$
\frac{C_{e q}}{q_{e}}=\frac{1}{K_{L} q_{m}}+\frac{C_{e q}}{q_{m}}
$$

$$
\log \mathrm{q}_{\mathrm{e}}=\log \mathrm{K}_{\mathrm{F}}+\frac{1}{n} \mathrm{C}_{\mathrm{eq}}
$$

$\mathrm{q}_{\mathrm{e}}=\mathrm{B} \ln \mathrm{K}_{\mathrm{T}}+\mathrm{B} \ln \mathrm{Ce}$

Where: $\mathrm{B}=\mathrm{RT} / \mathrm{b}$ and Ce: The equilibrium concentration $(\mathrm{mg} / \mathrm{L})$, qe: The amount adsorbed per gram of adsorbent $(\mathrm{mg} / \mathrm{g}), \mathrm{K}_{\mathrm{L}}$ : Langmuir constant $(\mathrm{L} / \mathrm{mg}), \mathrm{K}_{\mathrm{F}}$ : Freundlich constant $(\mathrm{L} / \mathrm{mg})$, $\mathrm{q}_{\mathrm{max}}$ : maximum adsorbed amount per gram of adsorbent $(\mathrm{mg} / \mathrm{g})$, $\mathrm{n}$ : Freundlich constant characteristic of the effectiveness of adsorbents towards given solutes, B: Temkin constant for heat of adsorption, $\mathrm{K}_{\mathrm{T}}$ : Adsorption equilibrium constant corresponding to the maximum binding energy[53].

The isotherm parameters $\mathrm{K}_{\mathrm{L}}, \mathrm{K}_{\mathrm{F}}, \mathrm{n}, \mathrm{K}_{\mathrm{T}}$ and $\mathrm{B}$ and correlation coefficients $\left(\mathrm{R}^{2}\right)$ for each model are regrouped in Table 2. 
Table 2. Parameters of the adsorption isothermsstudied for the A2 Nanocomposite

\begin{tabular}{ll}
\hline Isotherm model & A2 Nanocomposite \\
\hline Langmuir & \\
$\mathbf{q} \mathbf{~ m}(\mathbf{m g} / \mathbf{g})$ & 833 \\
$\mathbf{K}_{\mathbf{L}}$ & 0.77 \\
$\mathbf{R}_{\mathbf{L}}(\mathbf{L} / \mathbf{m g})$ & 0.92 \\
$\mathbf{R}^{\mathbf{2}}$ & 0.98 \\
\hline $\mathbf{F r e u n d l i c h}$ & \\
$\mathbf{K}_{\mathbf{F}}$ & 12.74 \\
$\mathbf{1} / \mathbf{n}$ & 0.27 \\
$\mathbf{R}^{\mathbf{2}}$ & 0.92 \\
\hline Temkin & \\
$\mathbf{B}$ & 106.96 \\
$\mathbf{K}_{\mathbf{T}}$ & 0.925 \\
$\mathbf{R}^{\mathbf{2}}$ & \\
\hline
\end{tabular}

$\mathrm{R}_{\mathrm{L}}$ is an important characteristic of the Langmuir isotherm which expressed a dimensionless constant, called equilibrium parameter and determined by the following equation:

$\mathrm{R}_{\mathrm{L}}=1 /$ racine $\left(1+\left(\mathrm{K}_{\mathrm{L}} * \mathrm{C}_{0}\right)\right)$

An equilibrium parameter $R_{L}>1$ shows that the adsorption is unfavorable, if $R_{L}=1$ the adsorption is in linear form, the adsorption can be favorable when $0<\mathrm{R}_{\mathrm{L}}<1$, and a zero separation factor $\left(\mathrm{R}_{\mathrm{L}}=0\right)$ indicates that adsorption is irreversible [42]. 


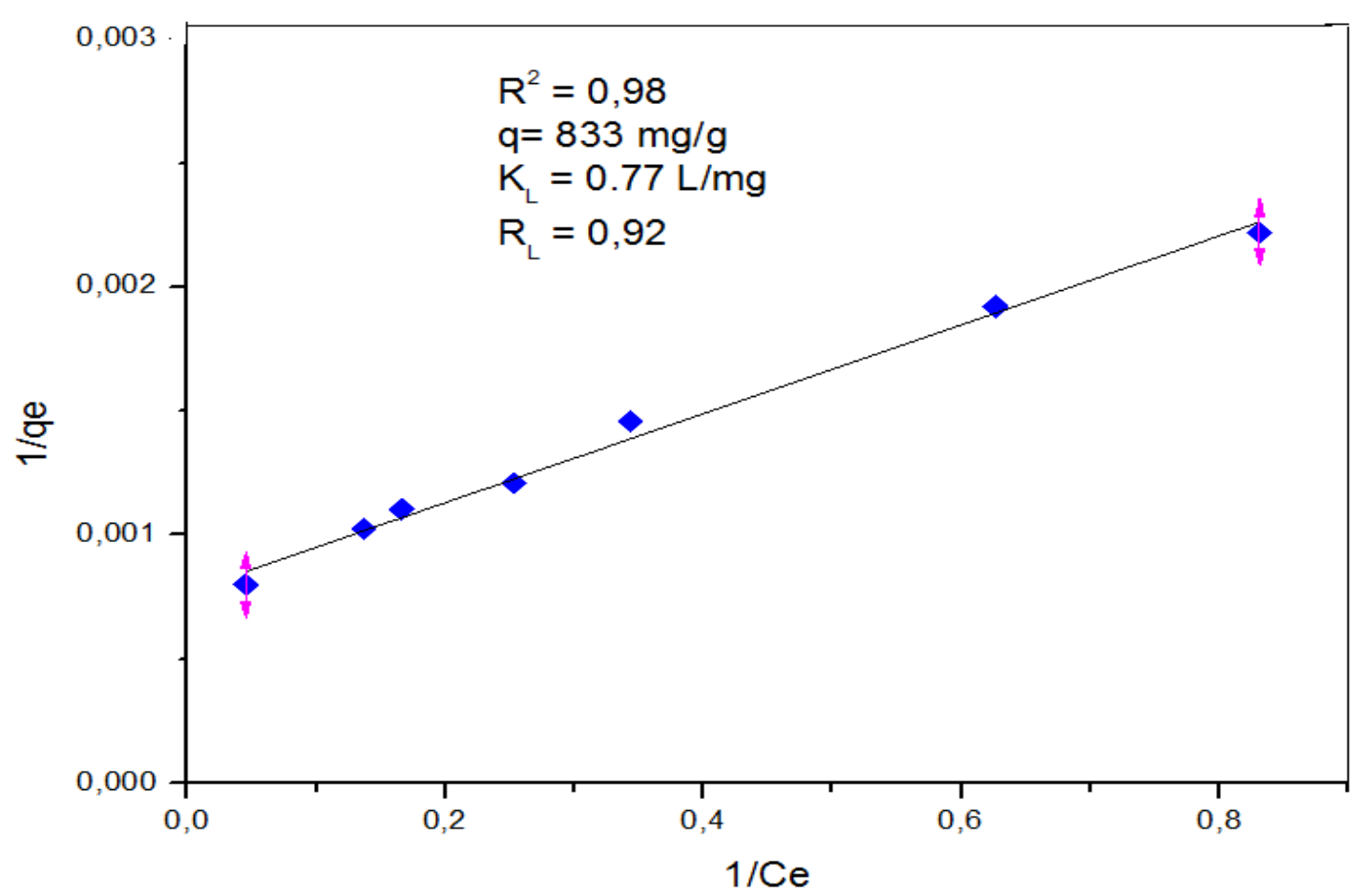

Figure 14. Transformed linear Langmuir isotherms for adsorption of MB on A2 Nanocomposite

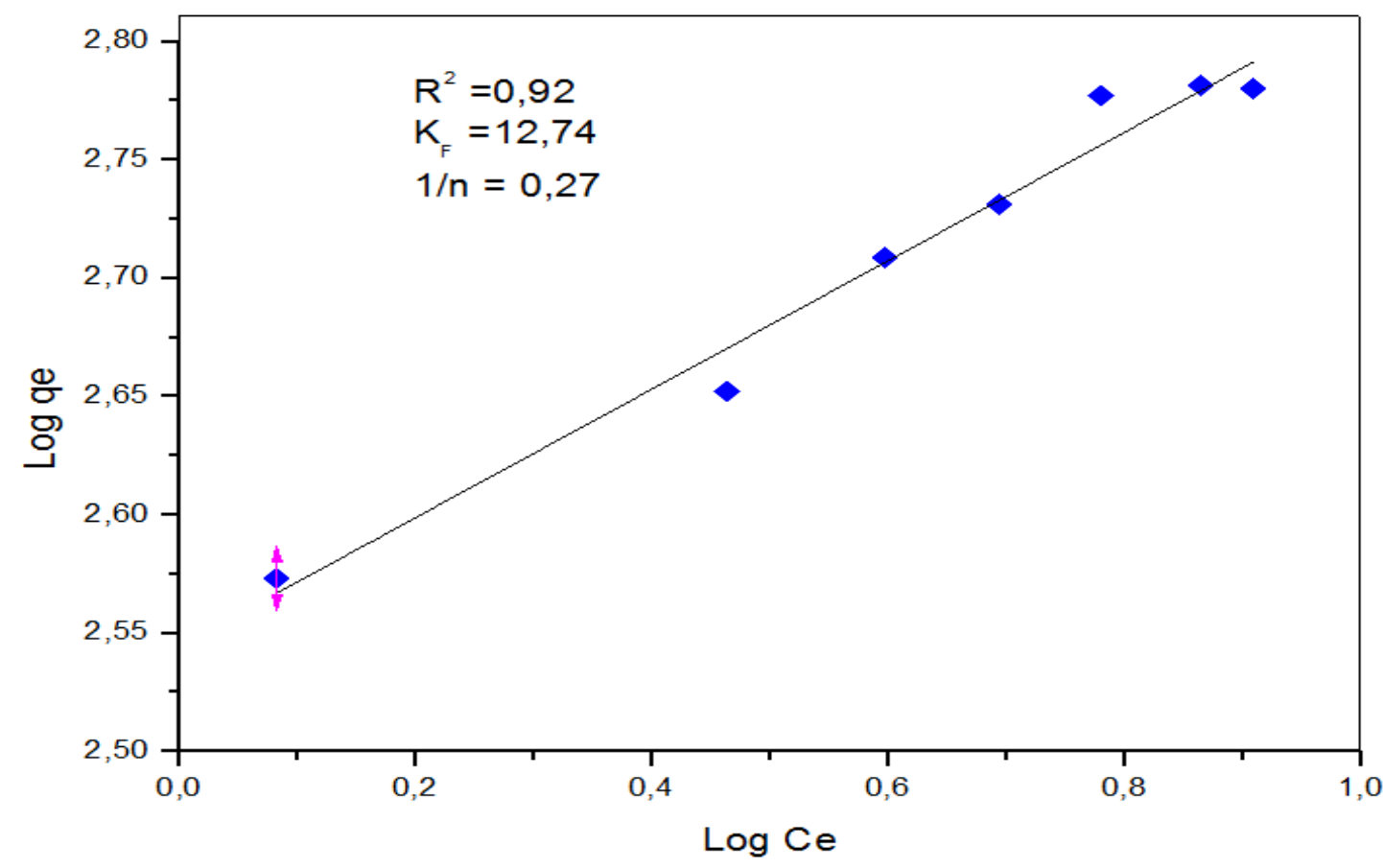

Figure 15. Transformed linear Freundlich isotherm for adsorption of MB on A2 Nanocomposite 


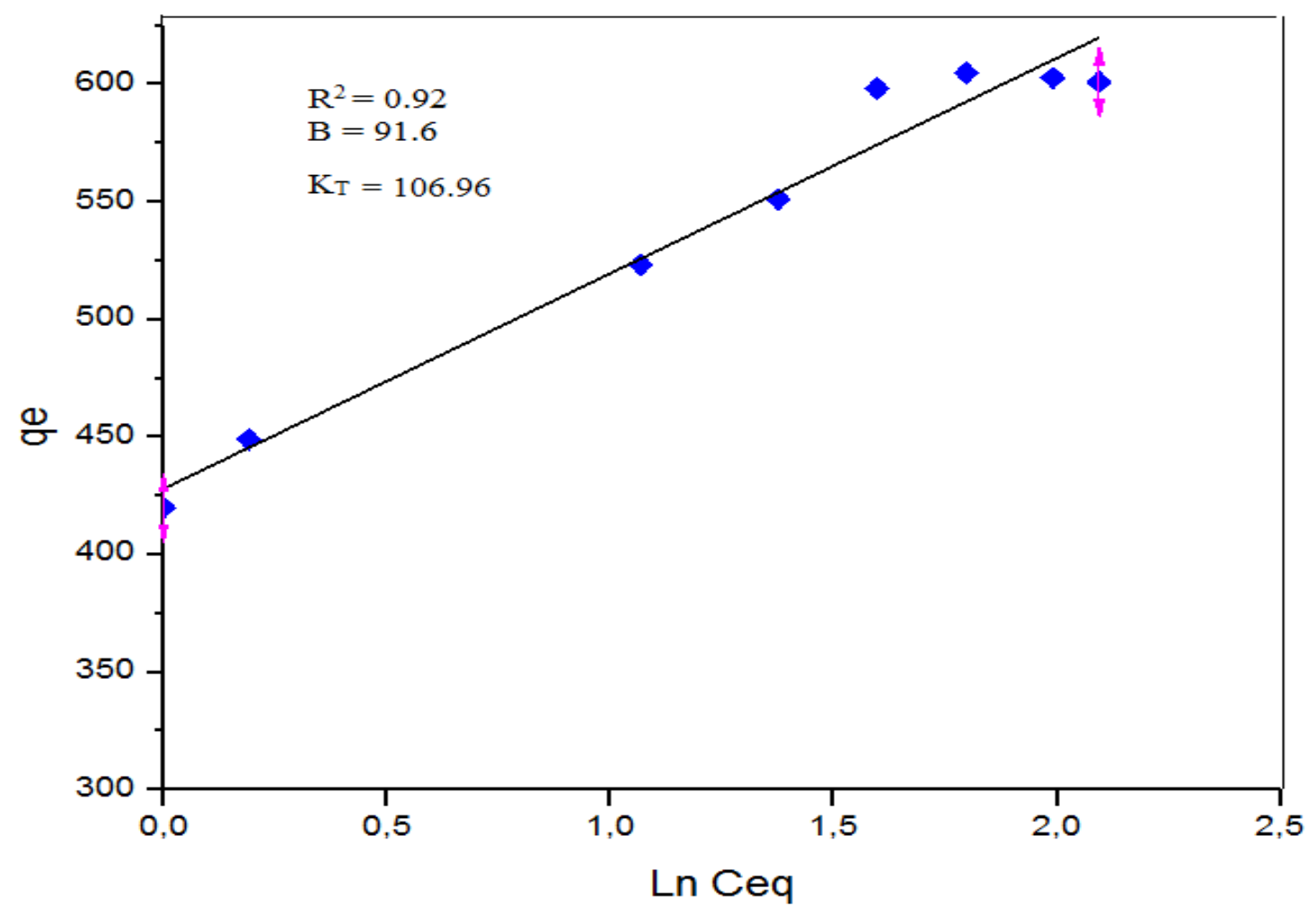

Figure 16. Transformed linear Temkin isotherm for adsorption of MB on A2 Nanocomposite

Based on the data obtained from Table 2. The application of the linearized forms of Langmuir, Freundlichand Temkin laws is made to verify which model is valid for modeling the adsorption isotherms in the interval of the studied concentrations. The obtained correlation coefficients $\left(\mathrm{R}^{2}=0.99\right)$ of Langmuir equations are much better to fit the experimental data than those of Freundlich and Temkin, indicating that the adsorption data were well described by Langmuir isotherm model and obeyed to monolayer molecule adsorption. In addition, the calculated data of equilibrium parameter $\mathrm{R}_{\mathrm{L}}$ from the Langmuir model were about 1 , indicating favorable adsorption of MB dye by the adsorbent.

In conclusion, the probable formation of a monolayer of MB dye homogeneously on surface of A2 nanocomposite, without any mutual interactions between localized sites whose energy is identical. Additionally, the maximum adsorption capacitiy for MB dye molecules by A2 nanocomposite given by Langmuir were about $800 \mathrm{mg} / \mathrm{g}$ Table 2, which were close to obtained experimental value. 
These results agreed with those previously reported for removal MB dye removal by sodium clay[54], mucuna beans[41], and microporous $\mathrm{Cu}$-doped BTC (Cu-BTC) metal-organic composite materials[42].

\section{Comparative study of $M B$ dye adsorption on different materials}

To assess the performance and adsorption efficiency of the synthesized nanocomposite based clay as an appropriate adsorbent for MB dye in aqueous solution is necessary to apply the in situ polymerization that polymer can be added to phyllosilicates by intercalation into the interfoliar space, and this leads thereafter to produce polymer/phyllosilicates nanocomposites with enhanced adsorptive properties.

The comparison of maximum adsorption capacities $(q \mathrm{e})$ of the MB dye on different types of adsorbent from aqueous solution was summarized in the Table 3. Overall, it can be noticed that this nanocomposite based clay is mainly low-cost adsorbent with different adsorptive properties; and could be used in large scale to remove successfully MB dye. The adsorption capacity of MB dye on A2 nanocomposite is higher compared with the other adsorbents such as activated carbon, chitosan-microspheres and different other types of clays Table 3. It can be suggested that A2 nanocomposite is a promising material for the efficient removal of dyes from aqueous solution. 
Table 3. Comparison of maximum adsorption capacity at equilibrium (qe) of nanocomposite A2 with different adsorbents

\begin{tabular}{lll}
\hline Adsorbent & qe(mg/g) & References \\
\hline Montmorillonite sodium (AS) & 240 & {$[43]$} \\
Montmorillonite calcium (AC) & 450 & {$[43]$} \\
Montmorillonite clay $\left(\mathrm{CC} 300^{\circ} \mathrm{C}\right)$ & 224 & {$[44]$} \\
Montmorillonite clay $\left(\mathrm{CC} 500^{\circ} \mathrm{C}\right)$ & 124 & {$[44]$} \\
Activated Carbon & 232.5 & {$[55]$} \\
Chemically modified GNPs & 225 & {$[56]$} \\
Activated carbon $(\mathrm{AC})$ & 476.88 & {$[57]$} \\
Chitosan-microspheres $(\mathrm{CMMS})$ & 371.7 & {$[58]$} \\
Fe ${ }_{3} \mathrm{O}_{4} @$ SiO & \\
microporous $(\mathrm{CH})_{3}-\mathrm{IL} / \mathrm{Talc}$ & 6.23 & {$[59]$} \\
metal-organic composite & 600 & {$[42]$} \\
Nanocomposite A2 & & This study \\
\hline
\end{tabular}

\section{Adsorption mechanism}

Based on the $\mathrm{pH}$ section, the adsorption mechanism of methylene blue dye is split into two phenomena Figure 17.

i)The hydrophobic interaction between the dye and the hydrophobic block (PCL) of the copolymer.

ii) The electrostatic interaction between the Si-O-, and Al-O- surface and edge groups of Bentonite and the sulfonic group of dye. 


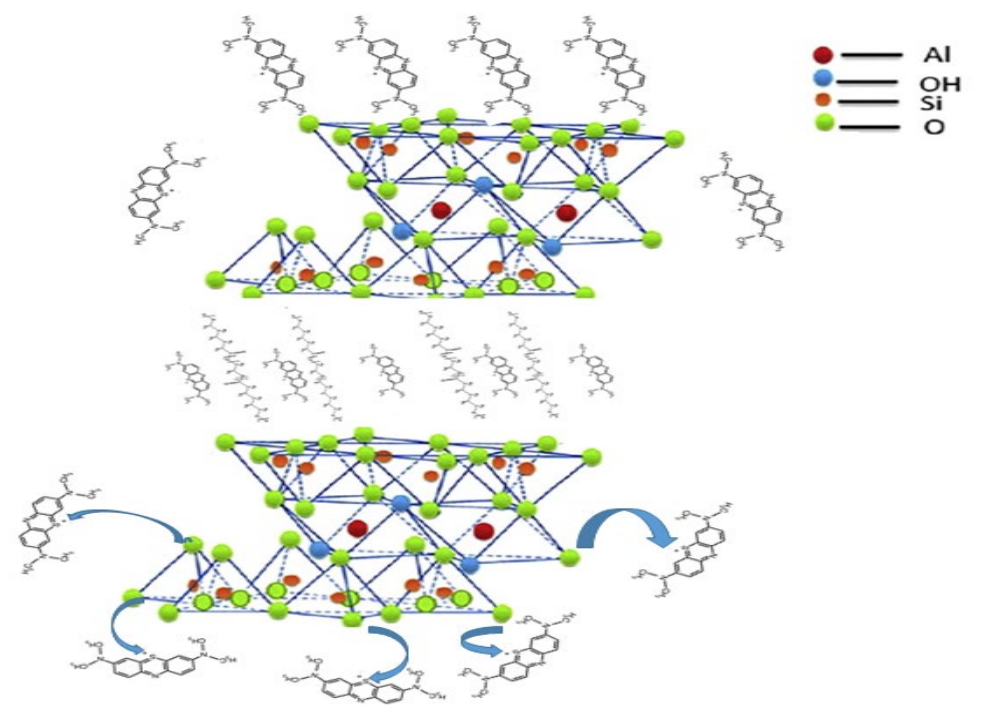

Figure 17. The possible interaction mechanism proposed between nanocomposite A2 and methylene bleu

\section{Conclusion}

PCL-PEG-PCL/Bentonite A2 nanocomposites have been prepared by in situ ring-opening polymerization of $\varepsilon$-caprolactone, in the presence of PEG chains, catalysed by a Bentonite. For this purpose, Bentonite- $\mathrm{Na}^{+}$has been exchanged by tetrabutylammonium cations. X-ray diffraction and transmission electron microscopy show the partially exfoliation of the silicate sheets in the PCL-PEG-PCL triblock copolymer/layered silicate nanocomposites having a low Alcontent ( 1 and $3 . \mathrm{wt} \%$ ). It was recognized that nanocomposite had the highest potential to improve crosslinking by adding 3.wt \% of BTH led to Good cure. The resulted A2 nanocomposites display good mechanical properties and a high adsorption capacity of dye. The maximum adsorption capacity of MB dye is about over $600 \mathrm{mg} / \mathrm{g}$ within $90 \mathrm{~min}$. It is also noticed that when the mass of A2 nanocomposite increased, the adsorption capacity of dye increases to reach an optimal value at $0.13 \mathrm{~g}$ of adsorbent in $\mathrm{pH}=6.8$ and ambient temperature. The Langmuir isotherm was found to be the most suitable model to represent the adsorption of the MB dye; the regression data show a good correlation between the Langmuir isothermal model and the obtained experimental results. For kinetic study, the experimental data fitted well with the pseudo-second order model for MB dye adsorption.

Acknowledgments The authors gratefully acknowledge the scientific support from Pr; Amina Ramdani, Department of Science, University Dr. Moulay Tahar, Saida Algeria. 


\section{Reference}

[1] J. N. Tiwari, K. Mahesh, N. H. Le, K. C. Kemp, R. Timilsina, R. N. Tiwari, K. S. Kim, "Reduced graphene oxide-based hydrogels for the efficient capture of dye pollutants from aqueous solutions," Carbon, vol. 56, pp. 173-182, 2013.

[2] G. Moussavi, M. Mahmoudi, "Removal of azo and anthraquinone reactive dyes from industrial wastewaters using $\mathrm{MgO}$ nanoparticles," J.Hazard.Mater, vol. 168, pp. 806-812, 2009.

[3] G. Moussavi, M. Mahmoudi,"Degradation and biodegradability improvement of the reactive red 198 azo dye using catalytic ozonation with MgOnanocrystals," Chem Eng, vol. 152, pp. 1-7, 2009.

[4] C. L. Young, J. K. Eui, Y. JiW, J. S . Hyun, "Removal of malachite green by adsorption and precipitation usingaminopropyl functionalized magnesium phyllosilicate," Journal of Hazardous Materials, vol. 192, pp. 62-70, 2011.

[5] A. Kadeche, A. Ramdani, M. Adjdir, A. Guendouzi, S. Taleb, M. Kaid, A. Deratani, " Preparation characterization and application of Fe-pillared bentonite to the removal of Coomassie blue dye from aqueous solutions," Research on Chemical Intermediates, vol. 46, pp. 4986-5008, 2020.

[6] D. Karadag, E. Akgul, S. Tok, F. Erturk, M. A. Kaya, M. Turan," Basic and Reactive Dye Removal Using Natural and Modified Zeolites," Chemical Engineering Data, vol. 52, pp. 2436-2441, 2007.

[7] M. Kute, H. Deo, S. Pai, "Adsorption of methylene blue using Ni/Fe layered double hydroxide," Pharma Innovat, vol. 7, pp. 190-192, 2018.

[8] A. Mishra, R. Srinivasan, M. Bajpai, R. Dubey, "Use of polyacrylamide-grafted Plantagopsyllium mucilage as a flocculant for treatment of textile wastewater," Colloid Polym Sci, vol. 282,pp. 722-727, 2004.

[9] M. Boudahri, D. Bouazza, M. Adjdir, H. Miloudi, N. Abdelkader, A. Tayeb, "Remediation of copper ions from aqueous solution using hybrid magadiite: kinetics, isotherm and mechanism of removal," Research. on Chemical Intermediates, vol. 44, pp. 6105-6117, 2018 . 
[10] Z. Cai, Y. Sun, W. Liu , F. Pan, P. Sun, J. Fu ," An overview of nanomaterials applied for removing dyes from wastewater," Environmental Science and Pollution Research, vol. 24, pp. 15882-15904, 2017.

[11] R. Cherrak, M. Hadjel, N. Benderdouche, M. Adjdir, A. Mokhtar, K. Khaldi, A. Sghier, P. G. Weidler, "Preparation of Nano-TiO2/Diatomite Composites by Non-hydrolytic Sol-Gel Process and its Application in Photocatalytic Degradation of Crystal Violet," Silicon, Vol. 12, pp. 927-935, 2020.

[12] M. Hamadanian, V. Jabbari, M. Shamshiri, M. Asad, I. Mutlay, "Preparation of novel hetero-nanostructures and high efficient visible light-active photocatalyst using incorporation of CNT as an electron-transfer channel into the support TiO2 and PbS," Journal Taiwan Inst Chem Eng, vol. 44, 748-757, 2013.

[13] M. Hamadanian, A. Akbari, V. Jabbari, "Electrospun titanium dioxide nanofibers fabrication, properties and its application in photo-oxidative degradation of methyl orange (MO)," Fibers Polym, vol. 12, pp. 880-885, 2011.

[14] T. Robinson, G. McMullan, R. Marchant, P. Nigam, "Remediation of dyes in textile effluent: a critical review on current treatment technologies with a proposed alternative," Bioresour. Technol, vol. 77, pp. 247-255, 2001.

[15] E. Van der Zee, S. Villaverde, "Combined anaerobic-aerobic treatment of azo dyes-a short review of bioreactor studies," Water Res, vol. 39, pp. 1425-1440, 2005.

[16] R. Gautam, A. S. Bassi, E. K. Yanful," A Review of Biodegradation of Synthetic Plastic and Foams " Appl Biochem Biotechnol, vol. 141, pp. 85-108, 2007.

[17] H. Chen, L. Kong, Y. Wang, " Enhancing the hydrophilicity and water permeability of polypropylene membranes by nitric acid activation and metal oxide deposition,"J. Membrane. Science, vol. 487, pp. 109-116, 2015.

[18] J. M. Raquez, Y. Habibi, M. Murariu, P. Dubois, "Polylactide PLA based nanocomposites," Prog.Polym.Sci, vol. 38, pp. 1504-1542, 2013.

[19] P. Bordes, E. Pollet, L. Averous, " Nano biocomposites Biodegradable polyester nano clay systems ," Prog.Polym.Sci, vol. 34, pp. 125-155, 2009. 
[20] M. Irani, H. Ismail, Z. Ahmed, "Preparation and propertiesof linear low density poly ethylene poly acrylic acid organo montmorillonite super absorbent hydrogel composites ," Polymer Testing, vol. 32, pp. 502-512, 2013.

[21] J. H. Chang, Y. U. An, G. S. Sur, " Poly lactic acid nanocomposites with various organo clays Thermo mechanical properties morphology and gas permeability," J. Polym. Phys. Appl. Sci, vol. 41, pp. 94-103, 2003.

[22] A. Oral, M. A. Tasdelen, A. L. Demirel, Y. Yagci, "Poly methyl methacrylate clay nanocomposites by photoinitiated free radical polymerization using intercalated monomer ," Polymer, vol. 50, pp. 3905-3910, 2009.

[23] M. A. Tasdelen, W. V. Camp, E. Goethals, P. Dubois, F. D. Prez, Y. Yagci, " Polytetrahydrofuran clay nanocomposites by in situ polymerization and clic chemistry processes," Macromolecules, vol. 41, pp. 6035-6040, 2008.

[24] M. Aydin, M. A. Tasdelen, T. Uyar, S. Jockusch, N. J. Turro, Y. Yagci, " Polystyrene clay nanocomposites by atom transfer radical nitroxide coupling chemistry," J. Polym. Sci. A, vol. 51, pp. 1024-1028, 2013.

[25] S. S. Ray, K. Yamada, M. Okamoto, Y. Fujimoto, A. Ogami, K. Ueda, "New polylactide/layered silicate nanocomposites Designing of materials with desired properties," Polymer, vol. 44, pp. 6633-6646, 2003.

[26] A. M. AlGhouti, Y. MAlDegs, "New adsorbents based on microemulsion modified diatomite and activated carbon for removing organic and inorganic pollutants from waste lubricants," J. Chemical. Engineering, vol. 173, pp. 115-128, 2011.

[27] M. Rafatullah, O. Sulaiman, R. Hashim, A. Anees, "Adsorption of methylene blue on low cost adsorbents ," J. Hazardous. Materials, vol. 177, pp. 70-80, 2010.

[28] C. Jiucun, W. Haodong, L. Wenqian, X. Jiming, Z. Lihui, S. Baoben, "Preparation of poly $\varepsilon$-caprolactone attapulgite nanocomposites via a combination of controlled ring-opening polymerization and click chemistry, " Colloid. Polym. Sci, vol. 288, pp. 173-179, 2010.

[29] Z. Zhang, L. Xu, P. Zhou, "Efficient removal of methylene blue using the mesoporous activated carbon obtained from mangosteen peel wastes: Kinetic, equilibrium, and thermodynamic studies, Microporous and Mesoporous Materials," Microporous and Mesoporous Materials Available, vol. 315, pp. 110904, 2021. 
[30] S. Haoue, H. Derdar, M. Belbachir, "Polymerization of ethylene glycol dimethacrylate (EGDM) using an Algerian clay as eco-catalyst (maghnite-Hp and Maghnite-Nap)," Bull. Chem. React. Eng. Catal, vol. 15, pp. 221-230, 2020.

[31] H. Moustafa, S. N. Lawandy, M. Rabee, "Effect of green modification of nanoclay on theadhesion behavior of EPDM rubber to polyester fabric, " J. Adhes. Adhes, vol. 100, pp. $102617,2020$.

[32] L. Mrah, R. Meghabar, " Influence du processus de modification de l'argile dans un nanocompositede silicate 'a couches de polypyrrole," J. Thermoplastic. composite. Materials, vol. 2, pp. $659,2020$.

[33] A. Harrane, M. Belbachir, "Synthesis of Biodegradable Polycaprolactone Montmorillonite Nanocomposites by Direct In-situ Polymerization Catalysed by Exchanged Clay ," Macro. mol. Symp, vol. 247, pp. 379-384, 2007.

[34] M. Zahraoui, A. Mokhtar, M. Adjdir, F. Bennabi, R. Khaled, A. Djelad, A. Bengueddach, M. Sassi, "Preparation of Al-magadiite material copper ions exchange and effect of counter-ions antibacterial and antifungal applications," Research on Chemical Intermediates, vol. 45, pp. 633-644, 2019.

[35] N. Bouchikhi, M. Adjdir, K. C. Bendeddouche, D. Bouazza,A. Mokhtar, F. Bennabi, A. Tabti, A. Sehmi, H. Miloudi, "Enhancement of adsorption capacity of low cost mesoporous MCM-41 and their antibacterial and antifungal activities," Materials. Research. Express, vol. 6, pp. 12, 2020.

[36] X. Weibing, G. Mingliang, H. Pingsheng, "Nonisothermal crystallization kinetics of polypropylene montmoriilonite nanocomposites," J. Polym. Sci, vol. 40, pp. 408-414, 2002.

[37] Q. Yuan, S. Awate, R. D. K. Misra, "Nonisothermal crystallization behavior of polypropylene clay nanocomposites," J. Eur. Polym, vol. 42, pp. 1994-2003, 2006.

[38] C. R. Tseng, H. Y. Lee, F. C. Chang, "Crystallization kinetics and crystallization behavior of syndiotactic polystyrene clay nanocomposites," J. Polym. Sci. Polym. Phys. B, vol. 39, pp. 2097-2107, 2001.

[39] F. Chivrac, E. Pollet, L. Avérous, "Nonisothermal crystallization behavior of poly butylene adipate co terephthalate clay nanobiocomposites," J. Polym. Sci. Polym. Phys. B, vol. 45, pp. 1503-1510, 2007. 
[40] M. Jouyandeh, S. M. R. Paran, R. Jannesari A. D. Puglia, M .RezaSaeb, "Protocol for nonisothermal cure analysis of thermoset composites," Prog. Org. Coat, vol. 131, pp. 333-339, 2019.

[41] M. Jouyandeh, S. M. R. Paran, A. Jannesari, M. R. Saebi, " Cure Indexfor thermoset composites," Progress. Organic . Coatings, vol. 127, pp. 429-434, 2019.

[42] M. Ş. A. Erena, H. Arslanoğlub, H. Çiftçi, "Production of microporous Cu-doped BTC (Cu-BTC) metal-organic framework composite materials, superior adsorbents for the removal of methylene blue Basic Blue 9," Journal. Environmental. Chemical. Engineering, vol. 8, pp. $104247,2020$.

[43] I. Fedal, A. Ramdani, S. Taleb, E. M. Gaigneaux, N. Batis, N. Ghaffour, "Adsorption capacity of methylene blue an organic pollutant by montmorillonite clay," Desalination. Water. Treatment, vol. 52, pp. 2654-2661, 2014.

[44] Y. L. Ma, Z. R. Xu, T. Guo, P.You, "Adsorption of methylene blue on Cu(II)exchanged montmorillonite," Colloid .Interface. Science, vol. 280, pp. 283-288, 2014.

[45] H. Cherifi, F. Bentahar, S. Hanini, "Kinetic studies on the adsorption of methylene blue onto vegetal fiber activated carbons," Applied. Surface. Science, vol. 282, pp. 52- 59, 2013.

[46] D. Kavitha, C. Namasivayam, "Experimental and kinetic studies on methylene blue adsorption by coir pith carbon," Bioresource. Technology, vol. 98, pp. 14-21, 2007.

[47] C. H. Weng, Y. F. Pan, "Adsorption of a cationic dye (methylene blue) onto spent activated clay," Journal of Hazardous Materials, vol. 144, pp. 355-362, 2007.

[48] Y. N. Liu, X. Zhou, X. Wang, K. Liang, Z. K. Yang, C. C. Shen, M. Imran, S. Sahar , A. W. Xu, "Hydrogenation/oxidation induced efficient reversible color switching between methylene blue and leuco-methylene blue," RSC . Advances, vol. 7, pp. 30080-30085, 2017.

[49] C. H. Giles, D. Smith, A. Huiston, "A general treatment and classification of the solute adsorption isotherm I Theoretical," J. Colloid. Interface. Sci, vol. 47, pp. 755-765, 1974.

[50] J. S. Piccin, L. C. S. Gomes, A. Feris, M. Gutterres, "Kinetics and isotherms of leather dye adsorption by tannery solid waste," J. Chemical. Engineering, vol. 183, pp. 30-38, 2012.

[51] A. Ramdani, Z. Taleb, A. Guendouzi, A. Kadeche, H. Herbache, A. Mostefai, S. Taleb, A. Deratani, "Mechanism study of metal ion adsorption on porous hydroxyapatite: experiments and modeling," Can .J. Chem, vol. 98, pp. 79-89, 2020. 
[52] A. Ramdani, A. Kadeche, M. Adjdir, Z. Taleb, D. khou, S. Taleb, A. Deratani, "Lead and cadmium removal by adsorption process using hydroxyapatite porous materials," Water. Practice. Technology, vol. 15 , pp. 130-140, 2020.

[53] J. Baliti, A. Asnaoui, S. Abouarnadasse, "L'élimination du bleu de méthylène par une argile naturelle de Taza en milieu aqueux," Journal. Innovative. Research. In. Advanced .Engineering, vol. 1, pp. 2149-2163, 2014.

[54] F. Tikhani, M. Jouyandeh, S. H. Jafari, S. Chabokrow, M. Ghahari, K. Gharanjig, F. Klein, N. Hampp, M. R. Ganjali, K. Formela, M. R. Saeb, "Cure Index demonstrates curing of epoxy composites containing silica nanoparticles of variable morphology and porosity," Progress in Organic Coatings, vol. 135, pp. 176-184, 2019.

[55] Y. Kuang, X. Zhang, S. Zhou, Water, "Adsorption of Methylene Blue in Water onto Activated Carbon by Surfactant Modification," vol. 587, pp. 2-19, 2020.

[56] R. M. Firdaus, N. I. M. Rosli, J. Ghanbaja, "Enhanced adsorption of methylene blue on chemically modified graphene nanoplatelets thanks to favorable interactions," J. Nanopart .Res, vol. 257, pp. 21, 2019.

[57] M. Peiyong, M. Meina, W. Jinzhou, Q. Yuzhi, W. Dongqiang, Z. Xianwen, "The effect of plastic on performance of activated carbon and study on adsorption of methylene blue," Journal of Materials Research, vol. 34, pp. 3040-3049, 2019.

[58] M. S. Jyothi, A. V. Jagadeesha, T. V. Kanakalakshmi, P. Mahesh, R. G. Balakrishna, S. Khantong, "Nanoparticles Impregnated Cross-Linked Porous Chitosan Microspheres for Efficient Adsorption of Methylene Blue from Pharmaceutical Waste Water," Journal of Polymers and the Environment, Vol. 27, pp. 2408-2418, 2019.

[59] A. Alizadeh, M. Fakhari, Z. Safaei, M. M. Khodeai, E. Repo, A. Asadi, "Ionic liquiddecorated $\mathrm{Fe}_{3} \mathrm{O}_{4} @ \mathrm{SiO}_{2}$ nanocomposite coated on talc sheets An efficient adsorbent for methylene blue in aqueous solution," Inorganic..Chemistry. Communications, vol. 121, pp. $108204,2020$. 
Figures

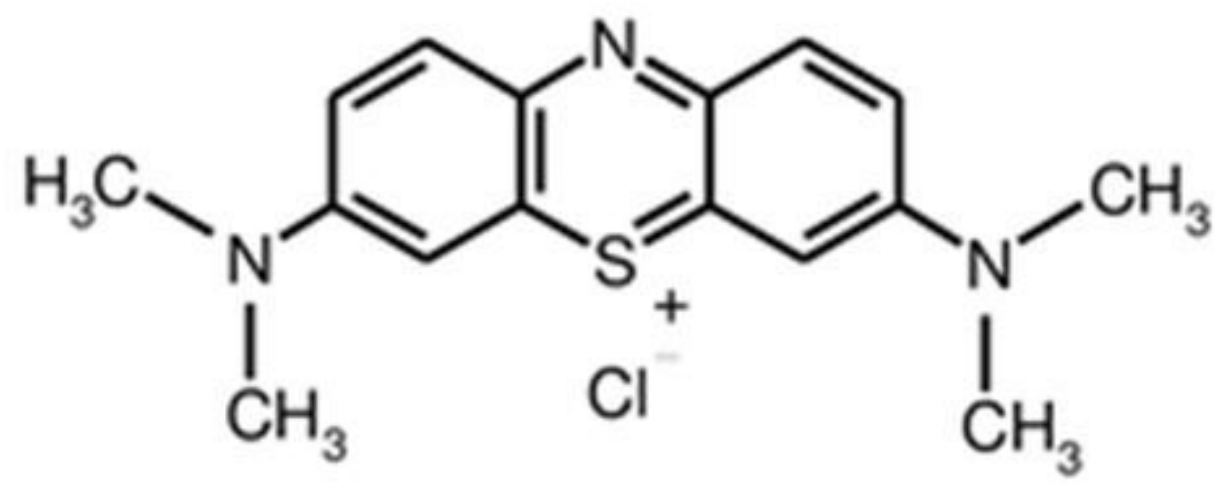

Figure 1

Structural formula of the MB dye

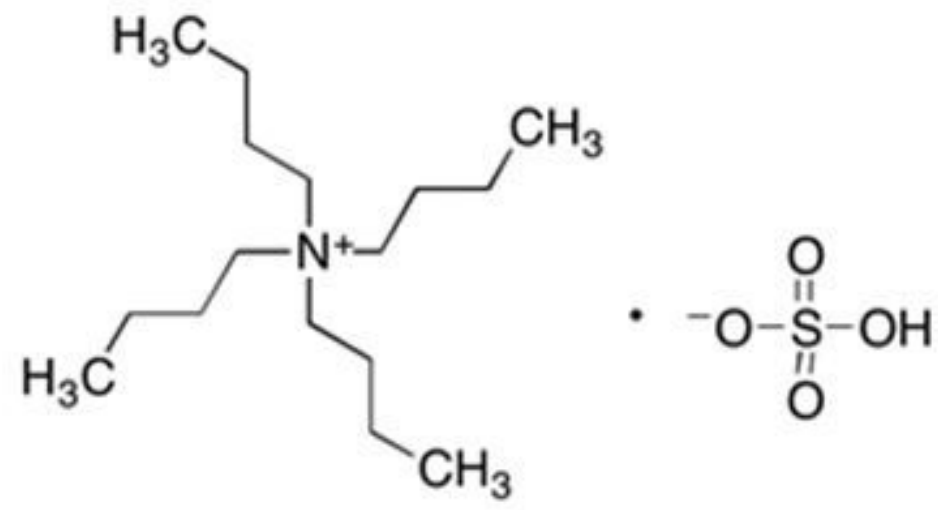

Figure 2

Structural formula of the TBHSA 


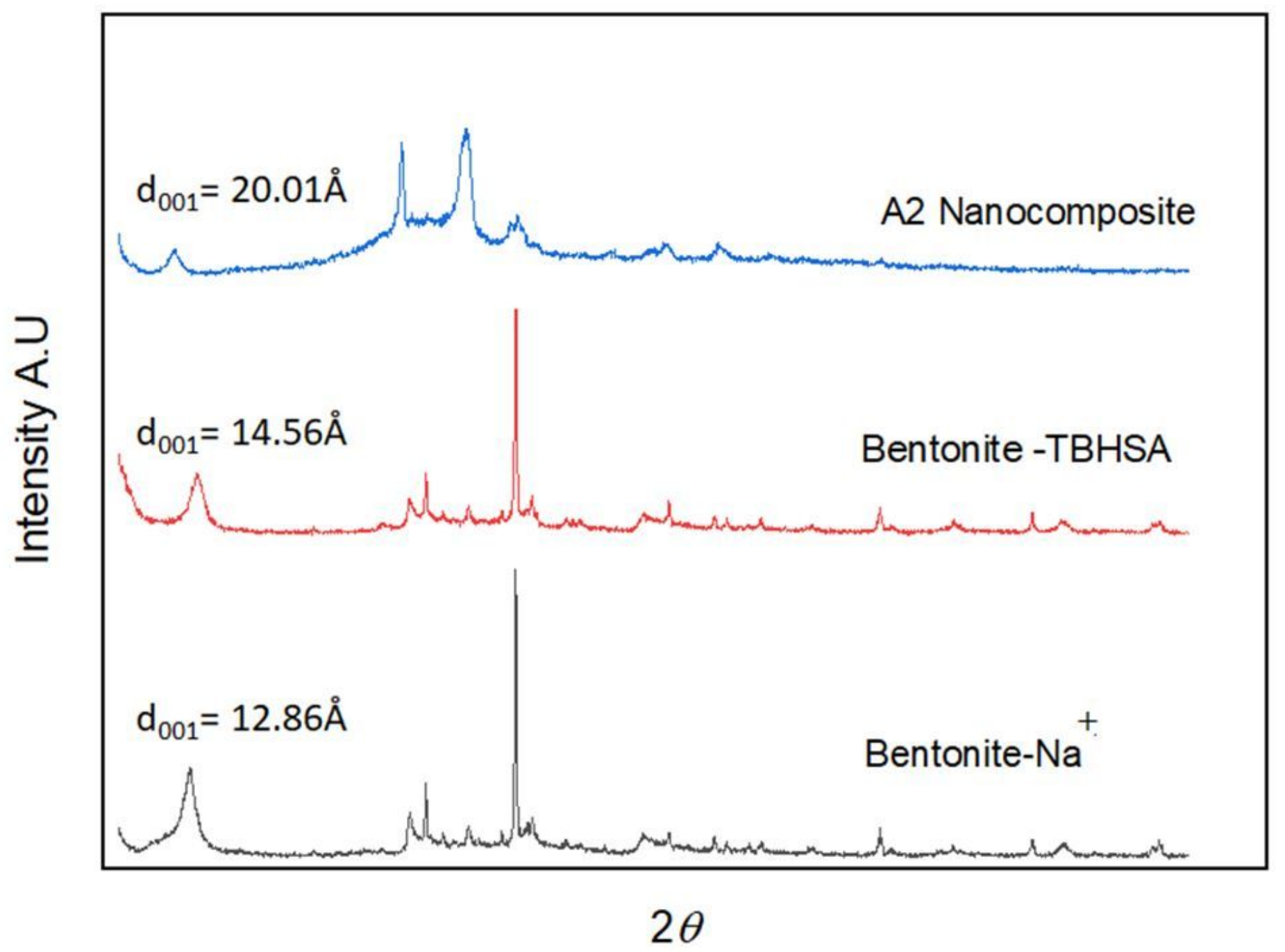

Figure 3

XRD patterns of Bentonite- Na+, Bentonite-TBHSA, and A2 Nanocomposite . 


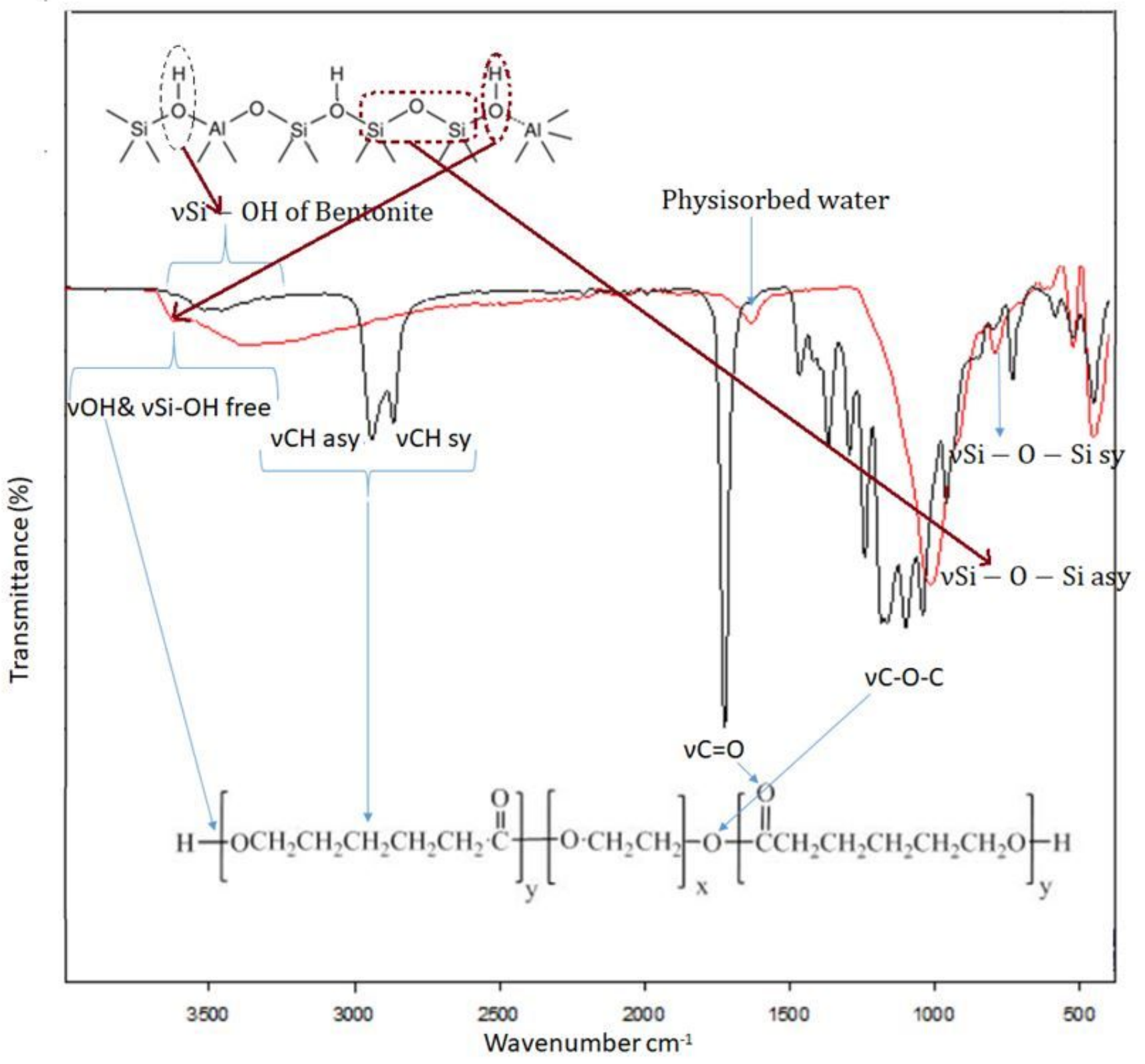

Figure 4

Exhibits the IR functional bands of Bentonite-Na+ and Nanocompsite A2. 


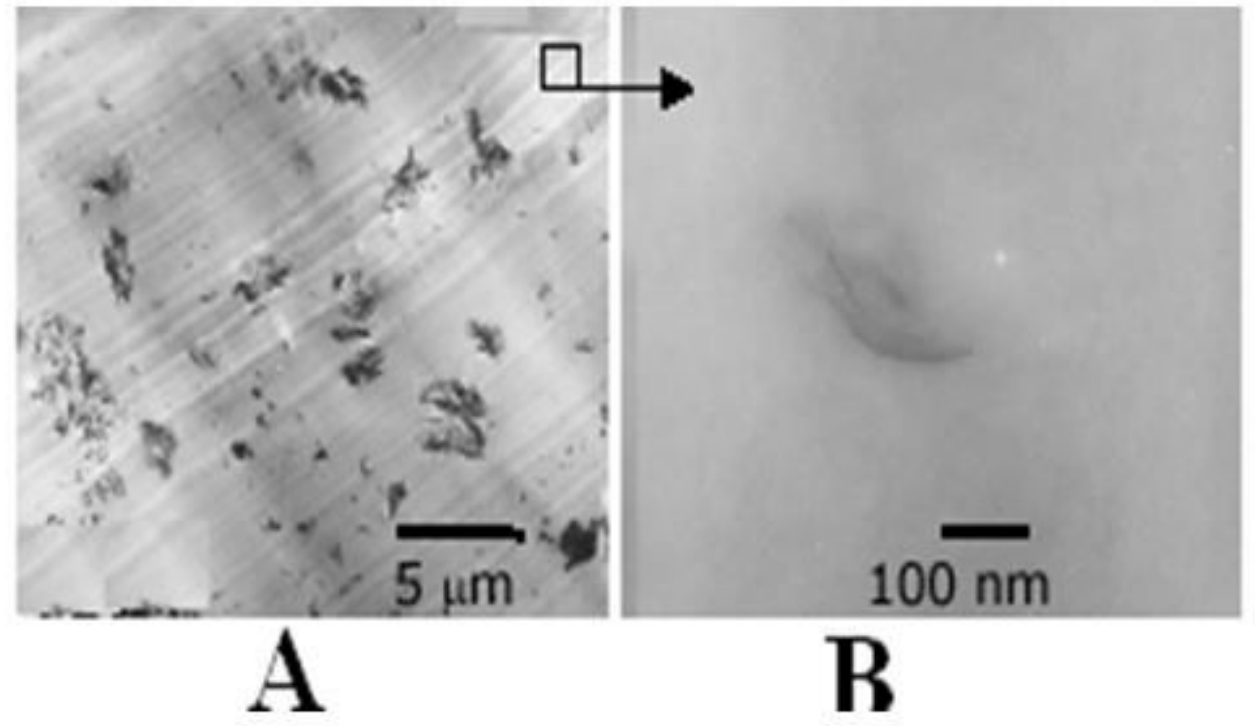

Figure 5

TEM microscopy images for A2 nanocomposite .

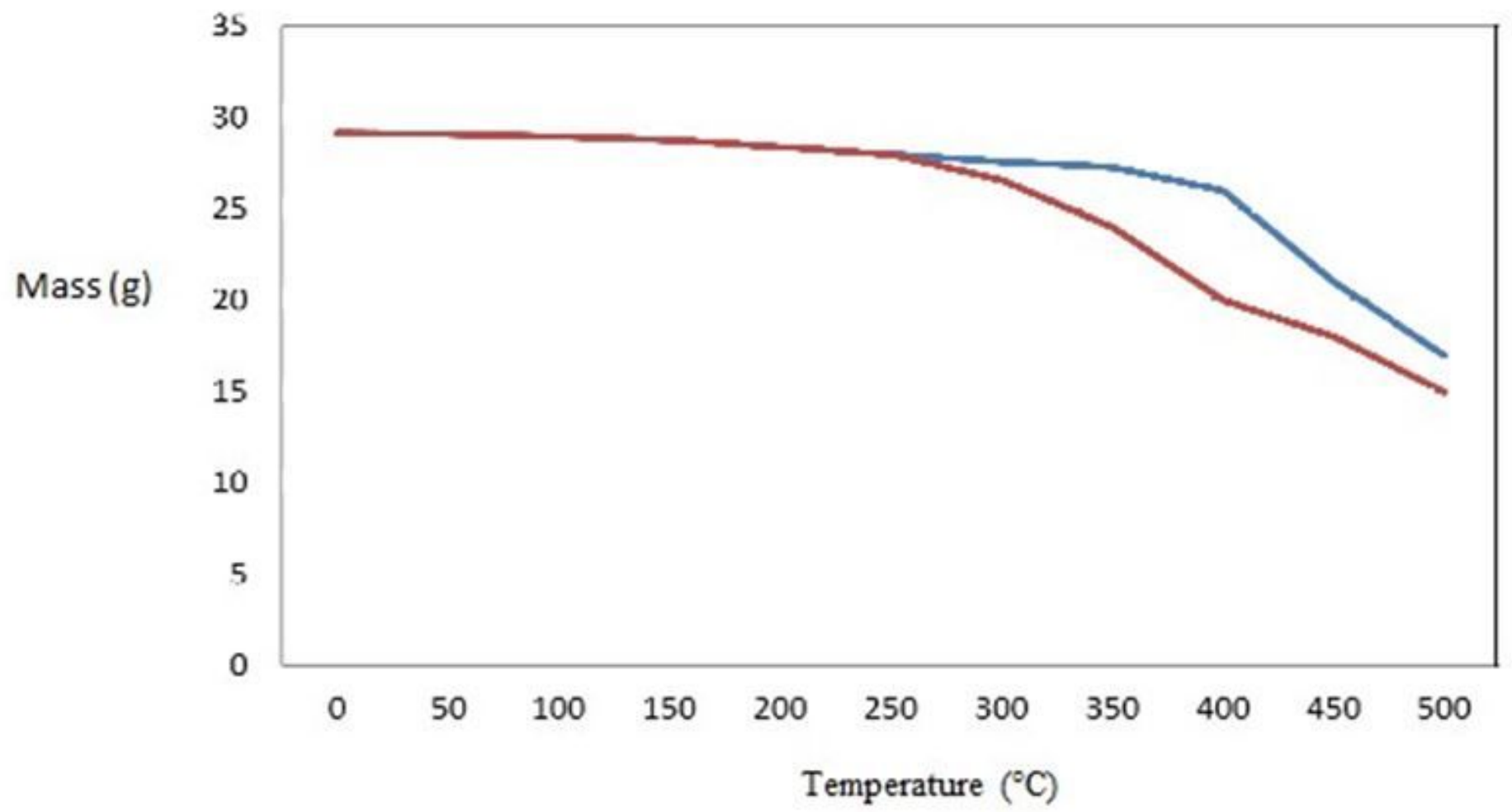

Figure 6

Thermogravimetrique Analyse of Nanocomposite A2- and copolymer pur - 


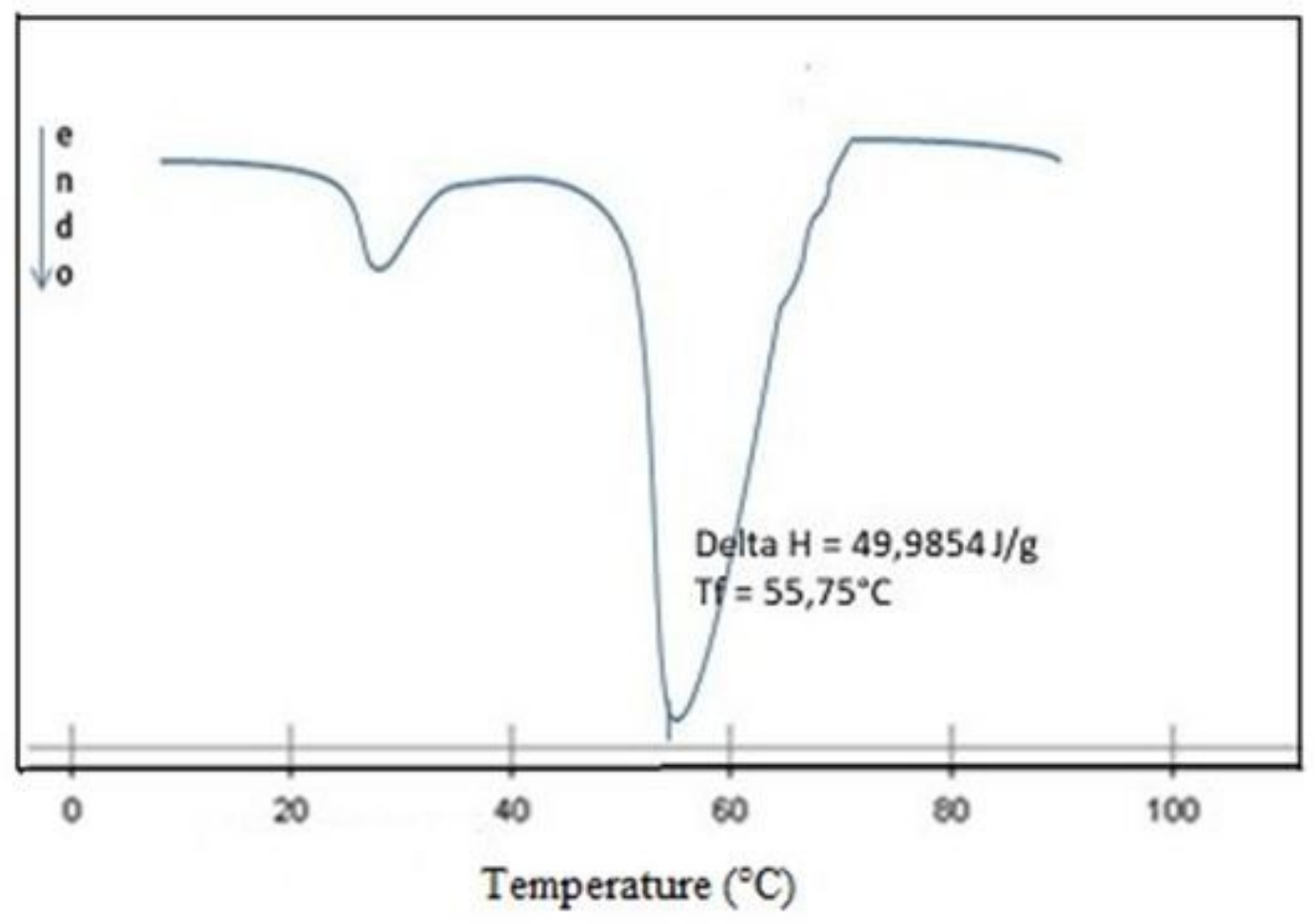

Figure 7

DSC Thermograms of A2 nanocomposites 


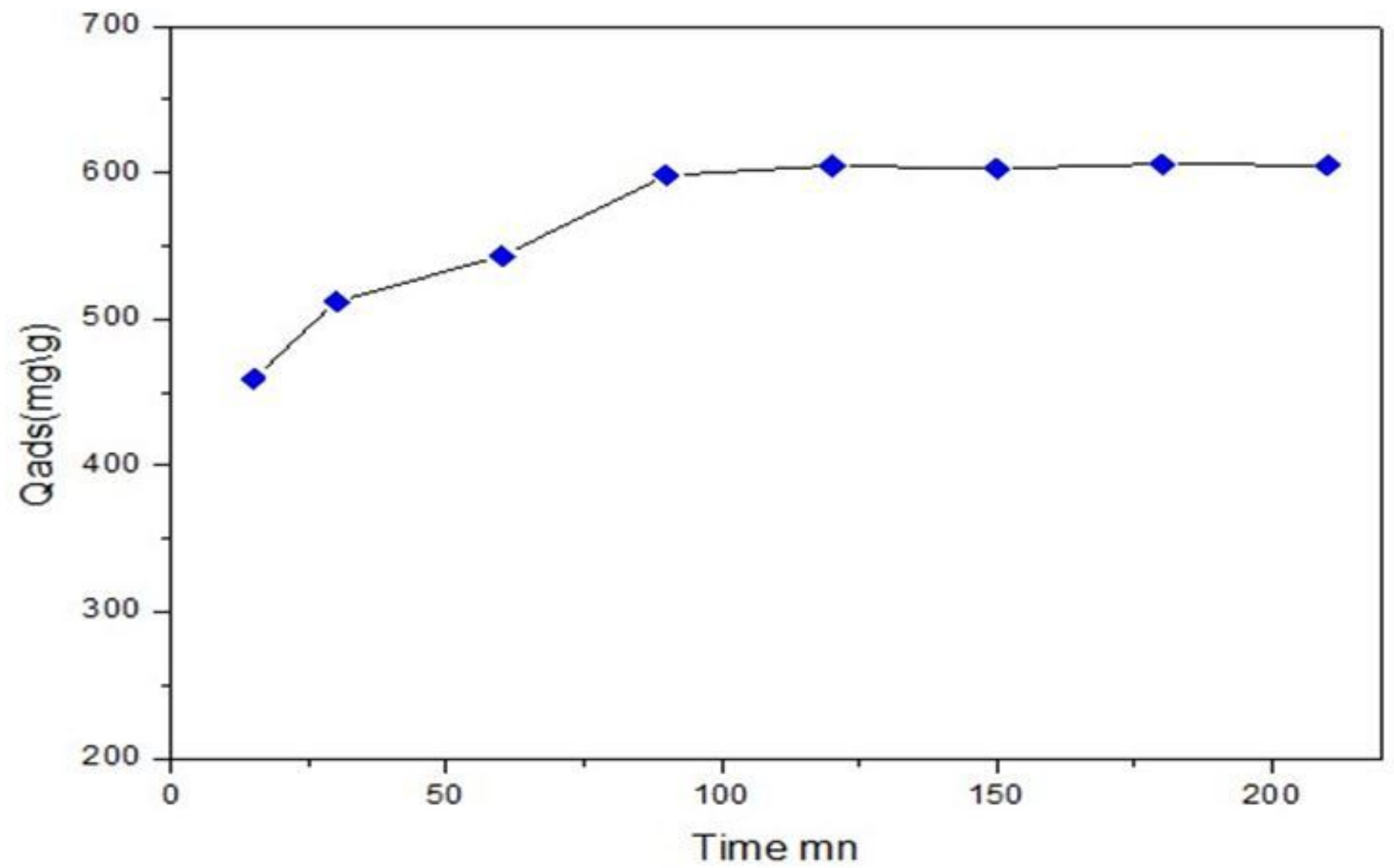

Figure 8

Kinetics of adsorption of $\mathrm{MB}$ on $\mathrm{A} 2$ nanocomposite 


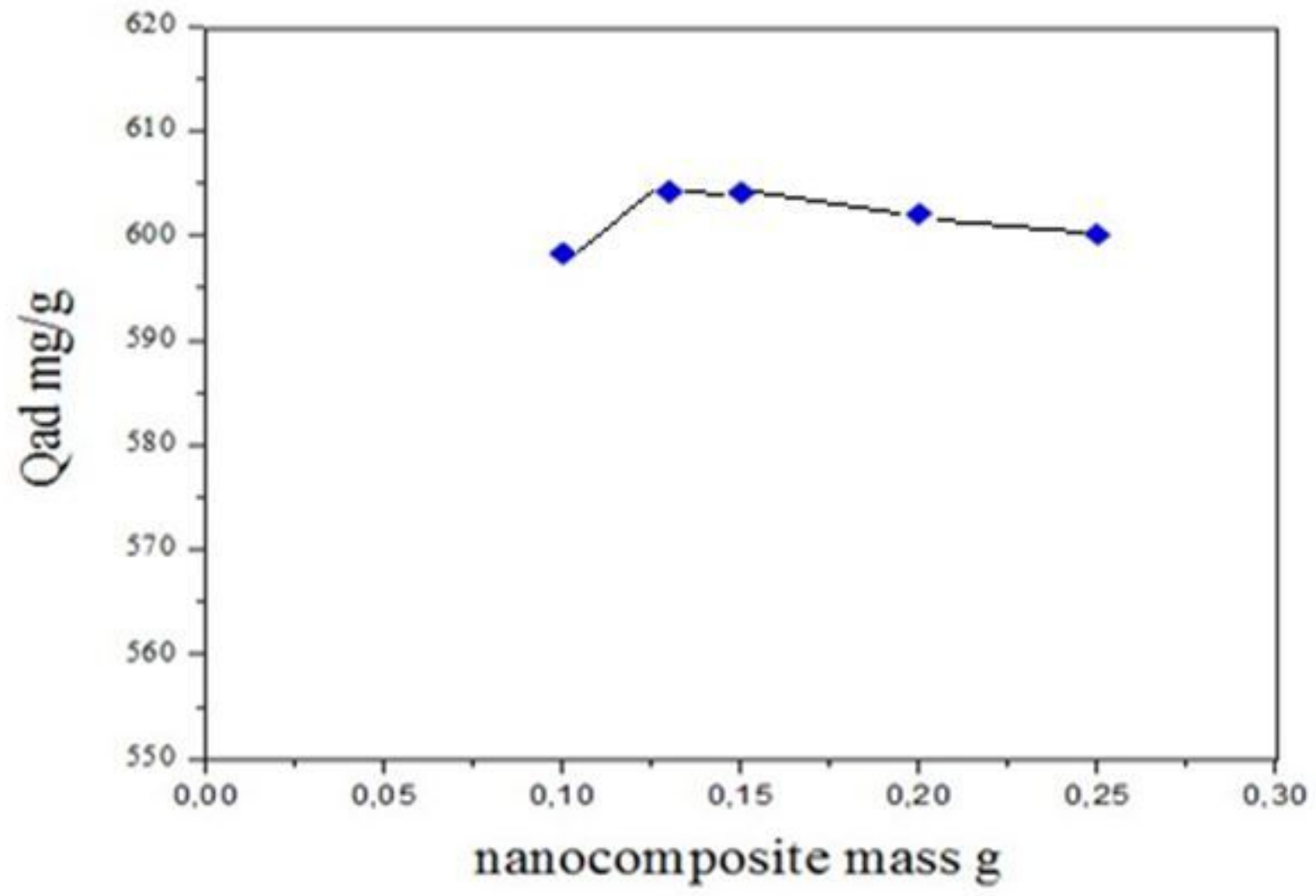

Figure 9

Effect of adsorbent mass on adsorption of MB 


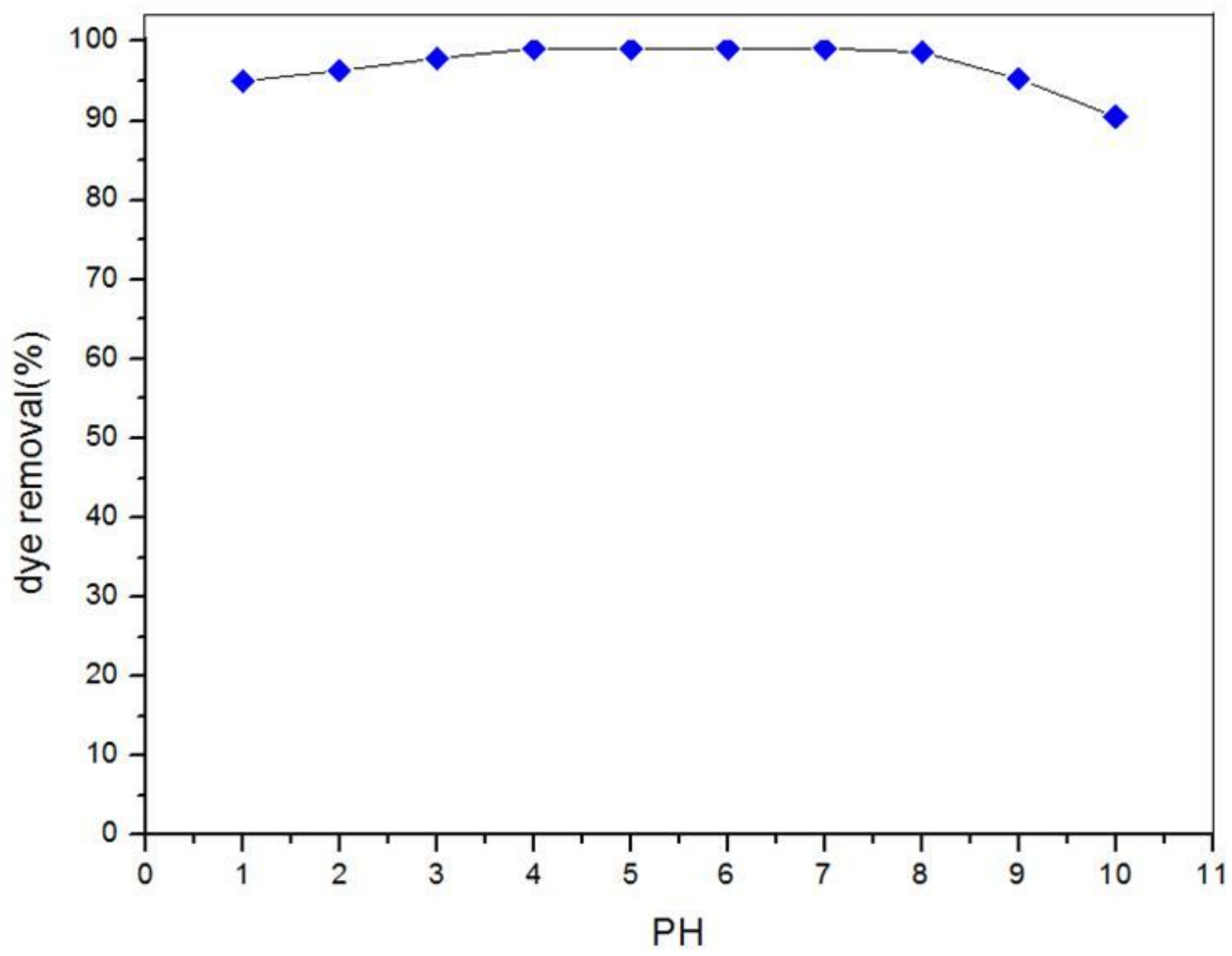

Figure 10

Effect of $\mathrm{pH}$ on adsorption of $\mathrm{MB}$ 


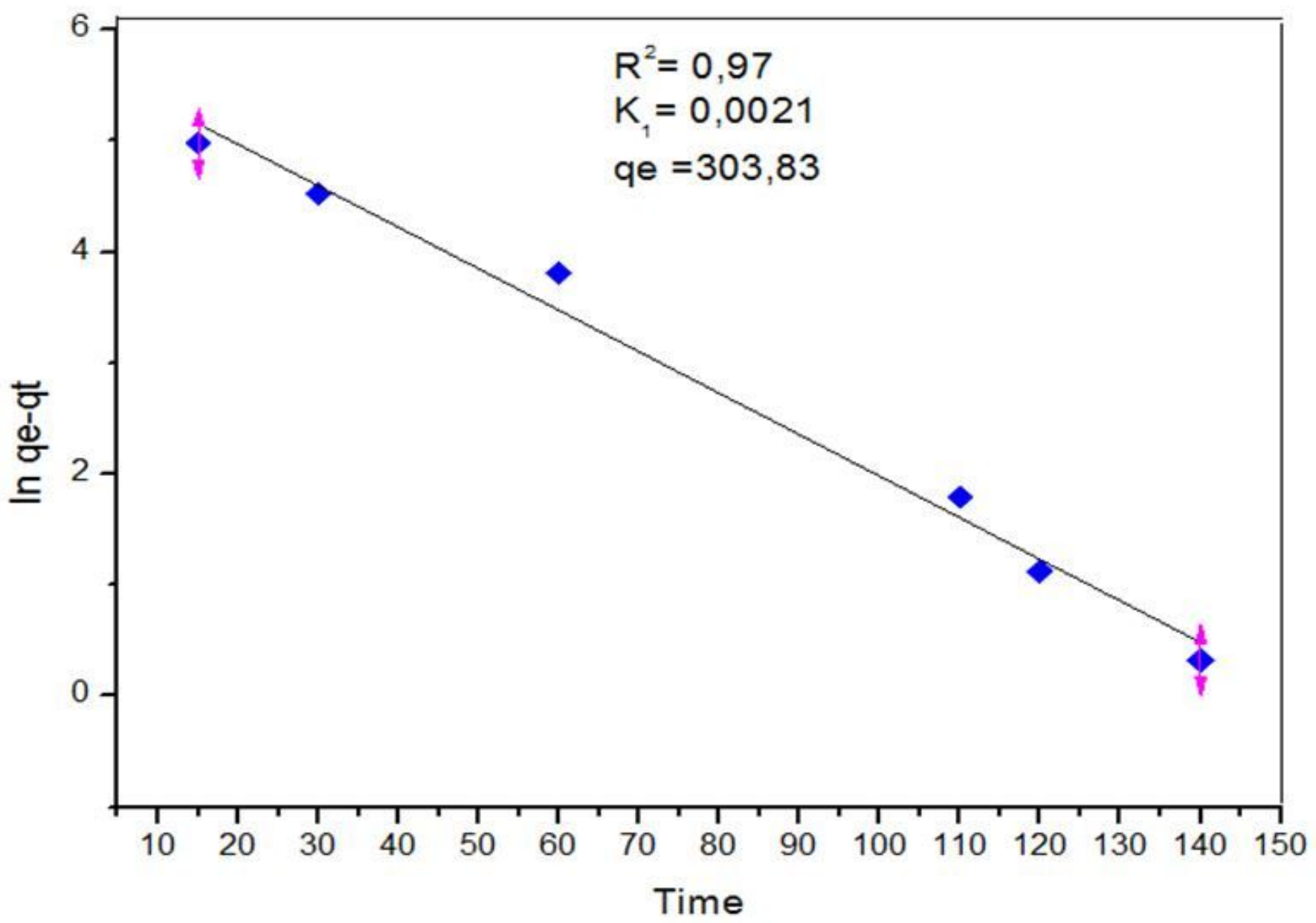

Figure 11

Pseudo-first order for the adsorption of MB on A2 nanocomposite 


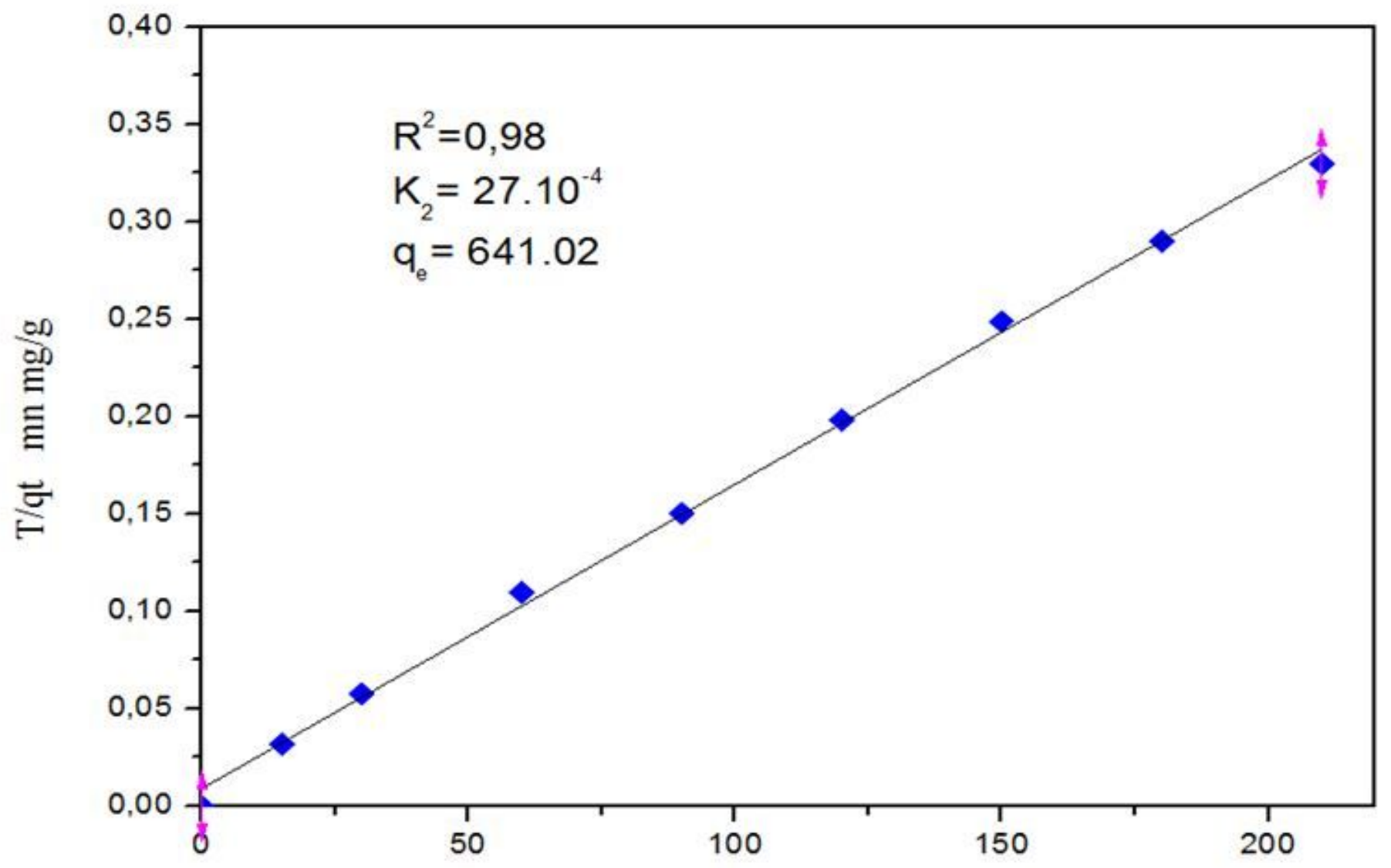

Time mn

Figure 12

Pseudo-second order for the adsorption of MB onto A2 nanocomposite 


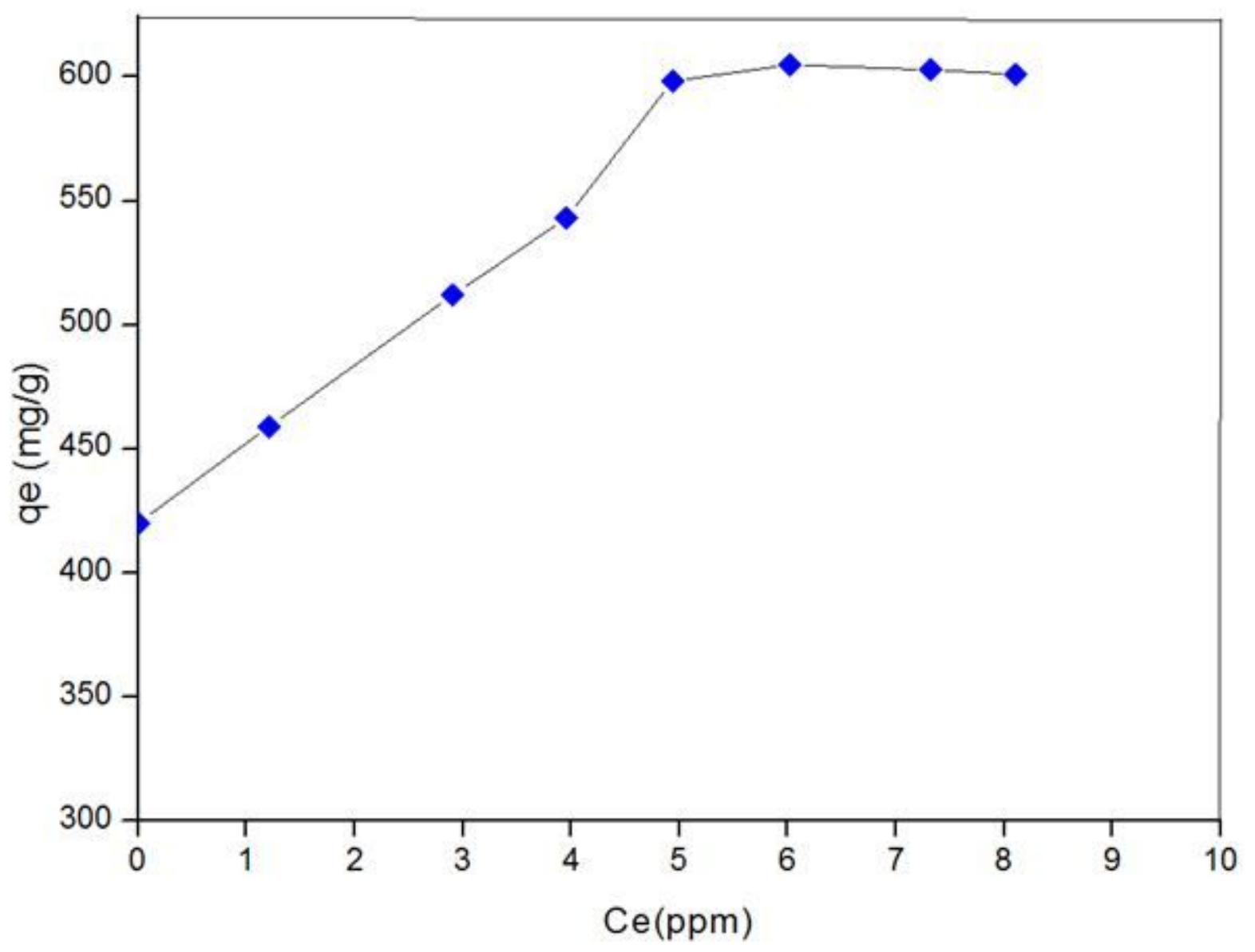

Figure 13

Adsorption isotherm of the MB onto $\mathrm{A} 2$ nanocomposite 


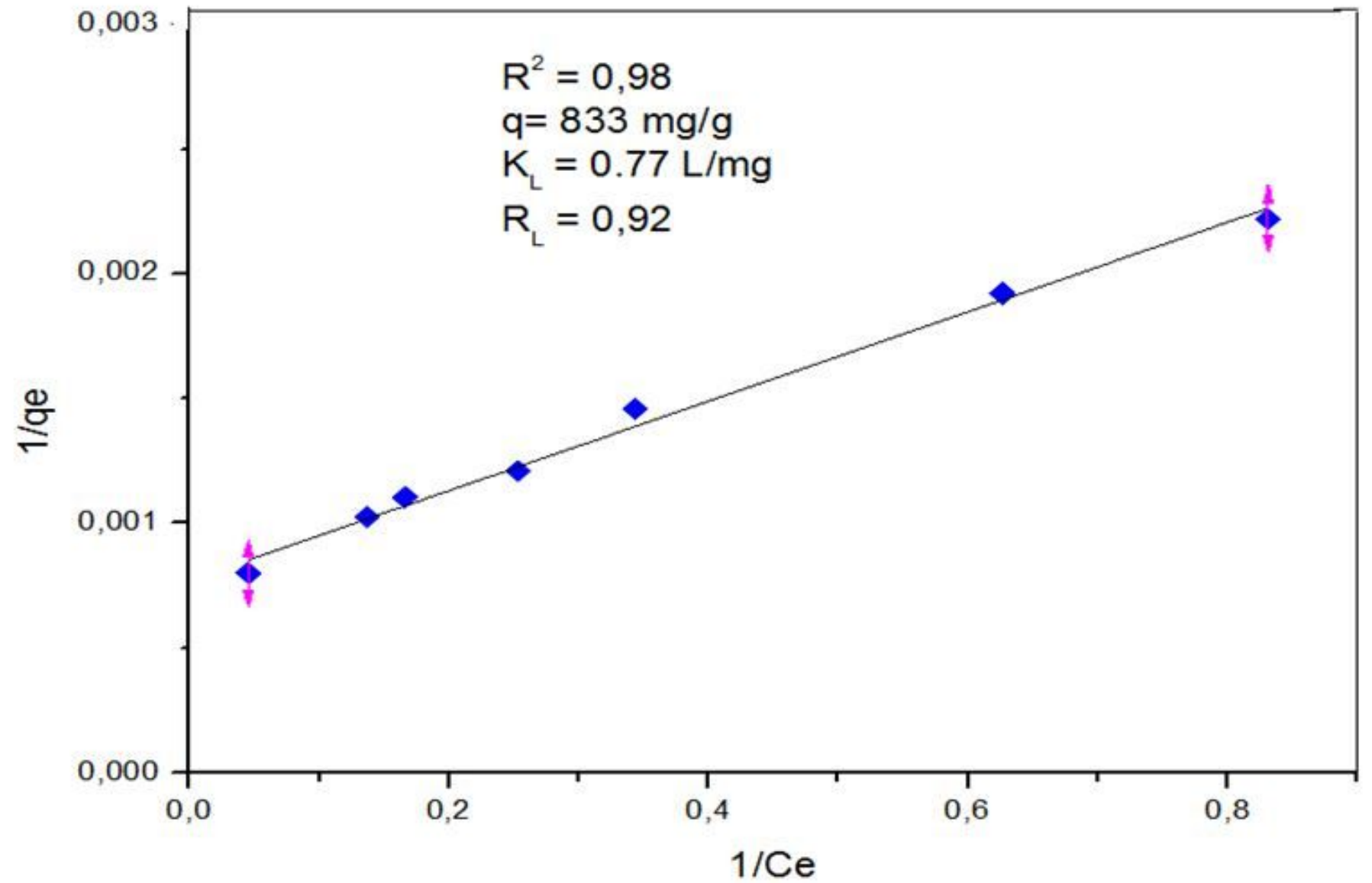

Figure 14

Transformed linear Langmuir isotherms for adsorption of MB on A2 Nanocomposite 


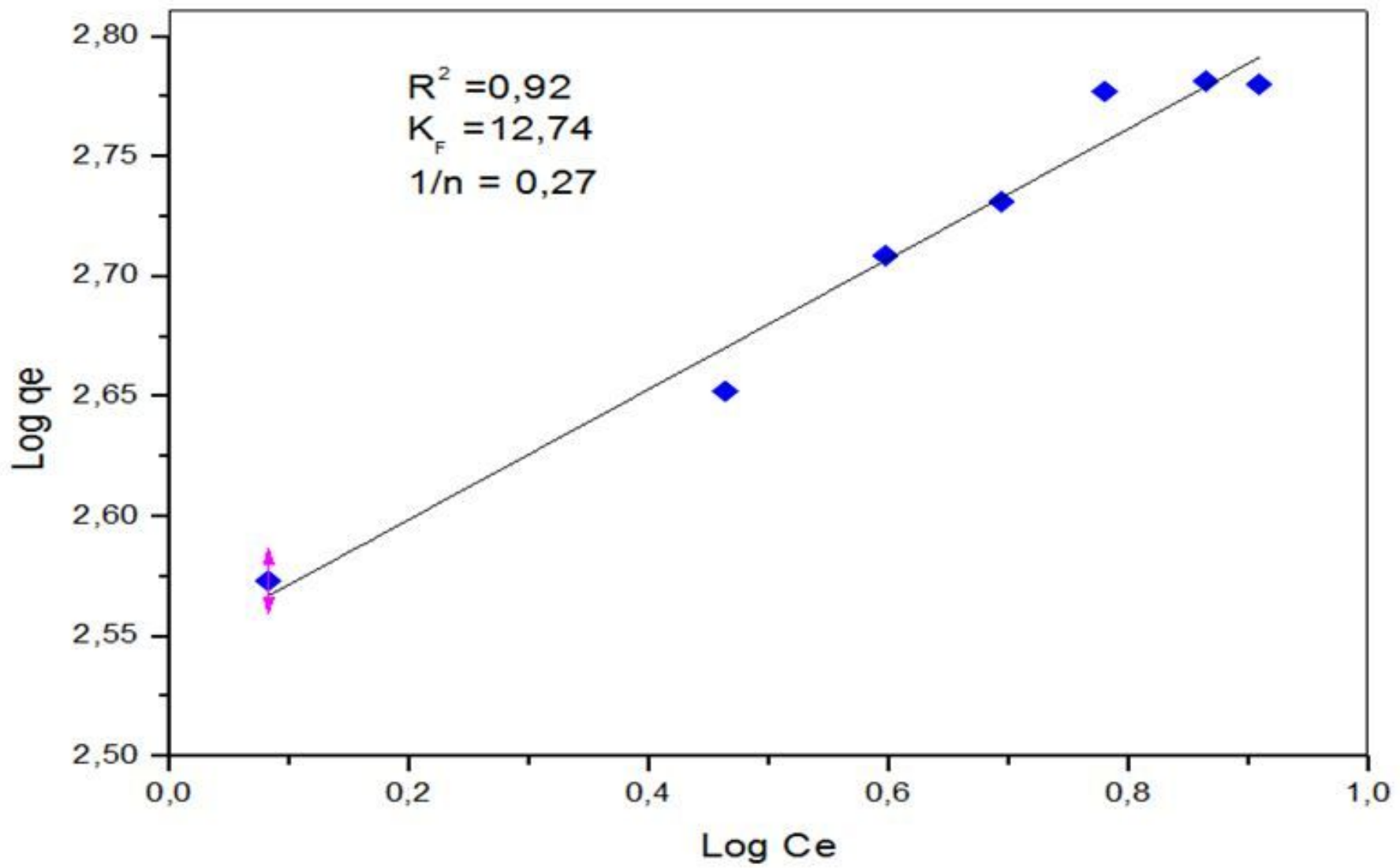

Figure 15

Transformed linear Freundlich isotherm for adsorption of MB on A2 Nanocomposite 


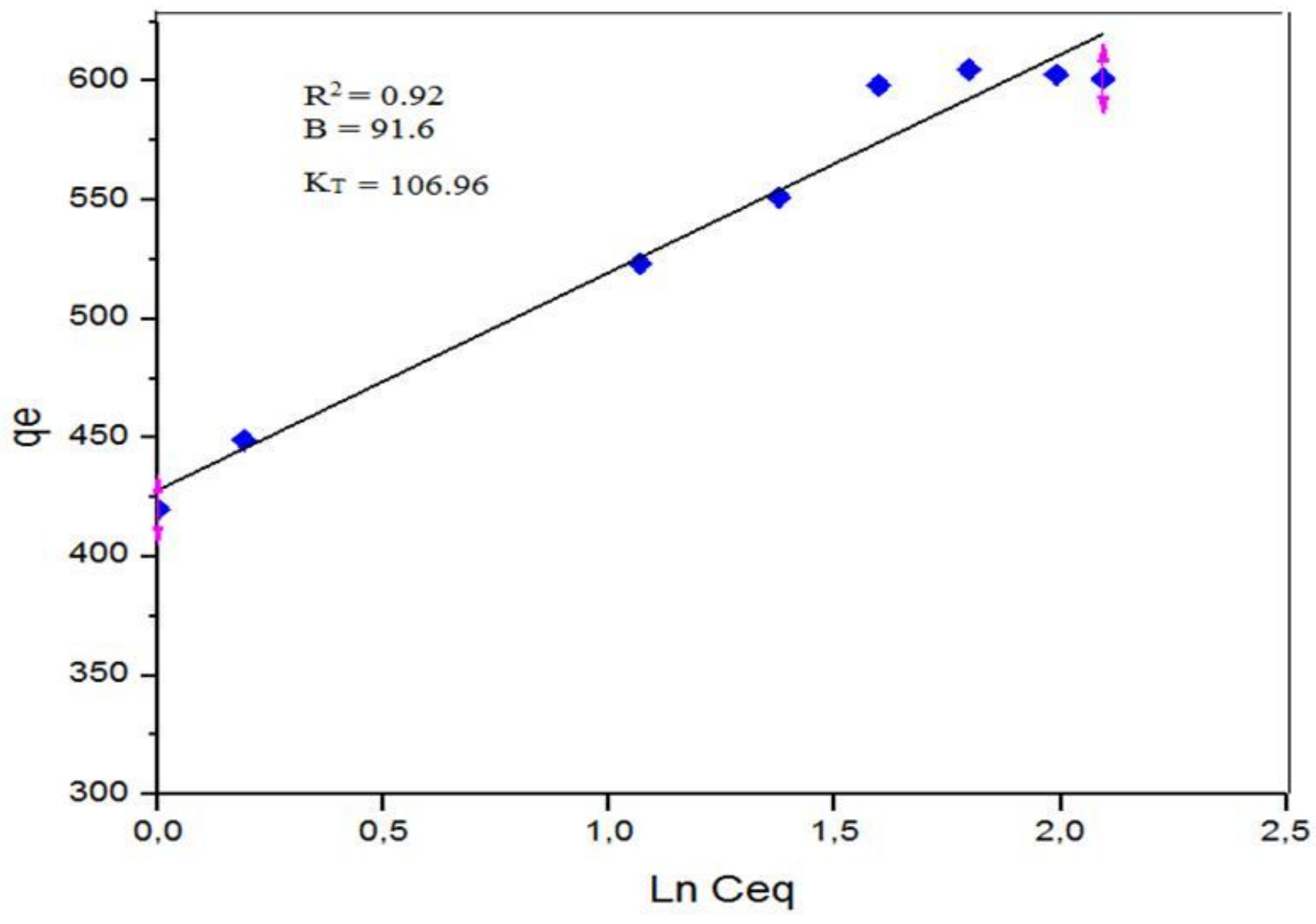

Figure 16

Transformed linear Temkin isotherm for adsorption of MB on A2 Nanocomposite 


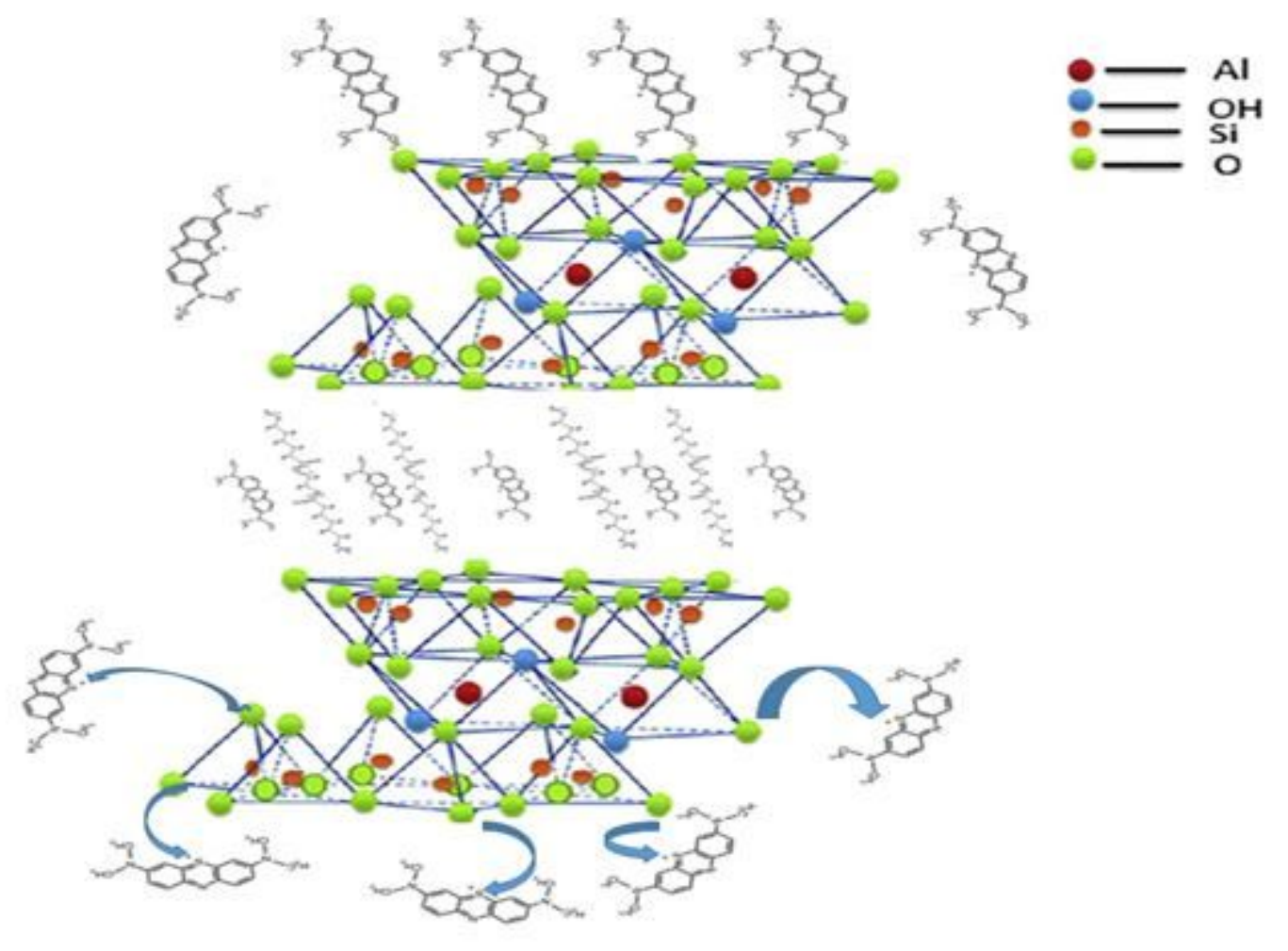

Figure 17

The possible interaction mechanism proposed between nanocomposite A2 and methylene bleu 